\title{
A numerical method for the simulation of low Mach number liquid-gas flows.
}

\author{
V. Daru ${ }^{(a, b)}$, P. Le Quéréa ${ }^{a}$ M.-C. Duluc ${ }^{(a, c)}$, O. Le Maître ${ }^{a}$ \\ ${ }^{\mathrm{a}}$ LIMSI-UPR CNRS 3251, BP 133, 91403 ORSAY Cedex, FRANCE \\ ${ }^{\mathrm{b}}$ Arts et Métiers ParisTech, DynFluid Lab. , 151 Bd de l'Hôpital, 75013 PARIS, \\ FRANCE \\ ${ }^{\mathrm{c}}$ CNAM, 292 Rue Saint Martin, 75141 PARIS Cedex 03, FRANCE
}

\begin{abstract}
This work is devoted to the numerical simulation of liquid-gas flows. The liquid phase is considered as incompressible, while the gas phase is treated as compressible in the low Mach number approximation. A single fluid two pressure model is developed and the front tracking method is used to track the interface. Navier-Stokes equations coupled with that of temperature are solved in the whole computational domain. Velocity, pressure and temperature fields are computed yielding a complete description of the dynamics for both phases. We show that our method is much more efficient than the so-called all Mach methods involving a single pressure, since large time steps can be used while retaining time accuracy. The model is first validated on a reference test problem solved using an accurate ALE technique to track the interface. Numerical examples in two space dimensions are next presented. They consist of air bubbles immersed in a closed cavity filled up with liquid water. The forced oscillations of the system consisting of the air bubbles and the liquid water are investigated. They are driven by a heat supply or a thermodynamic pressure difference between the bubbles.
\end{abstract}

Key words: Two-phase flow; front tracking; low Mach number flow; compressibility

\section{Introduction}

The numerical simulation of two-phase flow and related phenomena raises many different and difficult issues, from both modeling and computational standpoints. Several of these difficulties, such as dealing with the interface between two phases, tracking of the interface, implementing surface tension, 
coalescence, etc. are common to the simulation of flows of isothermal and incompressible multiphase fluids. Early attempts have made use of boundary fitted coordinates to simulate the flow around isolated bubbles of stationary or unsteady shapes [26]. These techniques allow one to impose very precisely the full interface conditions, but are limited to weak deformations and can hardly be extended to configurations involving many bubbles. These limitations have led to the development of Eulerian approaches, which have received much attention in the recent past and are still the subject of intensive ongoing research. Many types of numerical techniques have been developed to follow the interfaces, amongst which the VOF method [23, 37], the Level Set method $[42,38,43]$, the Front Tracking method [44, 40], interface capturing methods [1], and mixed methods [29]. A unified perspective of most of these methods can be found in [35].

One of the promising field of application of these techniques is the simulation of two-phase boiling flows that occur in confined environments. These simulations can be used to study various local dynamics processes and related heat transfer such as bubble growth or collapse, interaction between several bubbles, in the aim of deriving macroscopic interaction laws between phases used in more conventional modeling techniques, in which each of the two phases is defined as a local volume or mass fraction. One specific issue in the simulation of these boiling phenomena is the coexistence of a liquid and a gas phase in a non-isothermal environment, the gas being made up totally or partially of the liquid vapor. An additional difficulty arises in the case of confined flows, for which the variations of the thermodynamic pressure in the gaseous phase play an important role and must be taken into account. For instance, in situations like in a pressure cooker, it is the fact that the mean pressure in the vessel can rise above the atmospheric pressure that allows the liquid-vapor phase change to take place at temperatures well above $100^{\circ} \mathrm{C}$, improving the Carnot efficiency or reducing the cooking time. The present work is devoted to the design of such a model and its numerical implementation providing a way to give access to some value of the thermodynamic pressure.

One way to give access to the thermodynamic pressure is to treat all fluid phases as fully compressible. For high speed flows, this has promoted the development of explicit algorithms in which both the liquid and the gas phases are modeled with the fully compressible Navier-Stokes equations [20, 41, 36]. Let us also cite the work of Caiden et al [5], considering two-phase flow consisting of separate incompressible and high speed compressible regions. However, in many applications like those mentioned above, the velocities in the gas are very small, and the fully compressible approach raises different difficulties or issues of modeling or numerical nature. For low speed flows, one of these difficulties is linked to numerical representation of the pressure. Dynamic pressure variations corresponding to flow speeds of velocity $V$ are of order $\rho V^{2}$ which can be very small for flows of light low speed fluids and this raises the is- 
sue of finite precision arithmetic used in digital computers. For instance air flow velocities of $10^{-2} \mathrm{~ms}^{-1}$ correspond to differences in pressure of $10^{-4} \mathrm{~Pa}$, which cannot be represented in single precision arithmetic within a pressure field whose mean value is equal to the atmospheric pressure $\simeq 10^{5} \mathrm{~Pa}$, independently of the poor conditioning of the Jacobian matrix.

For fully compressible explicit methods, another major difficulty is linked to the severe limitation of the time step induced by the large value of sound velocity in the liquid. The speed of sound in a liquid can be more than $10^{4}$ or $10^{5}$ times larger than the convection velocity. For natural convection configurations where one has to integrate the equations on the order of a viscous or diffusion time scales, this limitation makes virtually impossible to attain the asymptotic flow regime. Alleviating this limitation leads one to promote an approach where the liquid is treated as completely incompressible. However, in many applications like those mentioned above, the velocities in the gas are very small, and the limitation of the time step based on the speed of sound (acoustic time step) in the gas remains severe. The so-called allMach or Mach-uniform methods that have received much attention in recent years are aimed at remedying this problem. These algorithms fall into two classes: density-based methods which we do not consider here as time accuracy is difficult to recover, and pressure based methods. The latter are derived from projection methods that are classically used for incompressible flows. They use an implicit algorithm for the calculation of pressure, which integrate the acoustic wave related part in the governing equations. Thereby the stability limitation of the time step due to acoustic propagation is avoided. Pressure-based methods also have the interesting property of being capable of handling both incompressible and compressible flows, as the pressure equation reduces to the usual Poisson equation when the Mach number tends to zero. This class of methods has been developed in several articles. Yabe et al. [47] utilize a Cubic Interpolated Polynomial (CIP) based time splitting predictor-corrector technique, separating advection and non-advection parts in the governing equations. Xiao [45],[46] brings several improvements to the method and proposes a conservative algorithm. Ida [16] incorporates into the CIP algorithm a multi-time step integration involving sub-iterations for solving the components of different time scales of the Navier-Stokes equations. In [18] a fully conservative method that uses a second order ENO method for the non oscillatory treatment of discontinuities is developed. The work in [22] presents a method quite similar but that rather uses a staggered MAC grid discretization.

Although this kind of methods may be thought of as a good candidate for our problem, the analysis in the literature shows that for unsteady calculations time steps of the order of the acoustic time step are generally used for accuracy reasons. In [16] is treated a case of bubble dynamics in an acoustic field, where it is shown that acoustics must be solved accurately for the entire flow accuracy, thus limiting the time step in the acoustic part of the solver to values close to the explicit value. The unsteady Oscillating Water Column test 
case is treated in [18] using an acoustic CFL number of 3 at most. In [22] a low Mach unsteady flow case is reported using again an acoustic CFL number of 3 for time accuracy of the solution. The conclusion that seems to emerge is that although the time step is not limited by a stability condition, it is still limited to values close to the explicit one for time accuracy reasons. This is also our finding, as we demonstrate below treating the Oscillating Water Column test case.

Although not subject to stability restrictions owing to their implicit nature, all Mach pressure based methods still consider the fully compressible model involving acoustics. However, for applications where the velocities in the gas are very small, a low Mach number model seems well adapted. In such an approach, acoustics is removed beforehand from the equations. The use of this approach was already proposed by [9] in the context of multicomponent gaseous flows, in the case of a potential approximation. Those methods were initially designed to handle low speed gas flows which can experience large variations of the mean pressure such as discharging flows from pressurized vessels [13] or natural convection flows due to very large temperature differences for which the Boussinesq approximation is no longer valid [33, 24]. In these methods the pressure is split into a mean pressure that can evolve in time and an additional component which is responsible for satisfying the continuity equation. This pressure splitting inhibits the local coupling between pressure and density, thus avoiding the simulation of acoustic waves and alleviating the corresponding stability criteria. Indeed, low Mach number approaches have been shown to be much more efficient and reliable than fully compressible models for the computation of natural convection flows in closed cavities $[25,32]$.

Another question to be addressed concerns the appropriate description of the dynamics of the flow within the gas phase. One strategy is to neglect the flow within each gaseous inclusion, which is then just described by its shape, mean pressure and temperature. Such an approach was followed by Caboussat et al $[4,3]$ in the isothermal case to handle mould filling. The case of bubbles with uniform but time dependent temperatures was largely investigated by Prosperetti's group, considering a gas bubble in a small tube [14] or a vapour bubble in a micro-channel [48]. The physical description considers uniform properties in the bubble (pressure, temperature, density). The liquid description is performed using an approximate potential flow [30] or the complete Navier-Stokes equations [31]. At the interface, the connection with bubble properties is achieved through standard jump equations while the numerical description performed in [31] makes use of free surface techniques.

Neglecting the gas dynamics may result in the impossibility to describe physical phenomena such as thermal convection effects for instance. In the microfluidic context, some operations like pumping or mixing rely on the gas 
dilatability to impose the liquid motion. We have shown that in such configurations the complete description of the flow in the gas is indeed necessary $[7,10]$.

In this work we report the development and numerical implementation of a physical model dedicated to the simulation of low speed non isothermal twophase flows in closed vessels. To be specific, amongst the type of configurations we have in mind we can list a steam engine or a pressure cooker, or microfluidic devices like thermopneumatic actuators. To this aim, we propose a numerical algorithm in which both the liquid and gaseous phases are governed by their corresponding momentum and energy equations, the liquid phase being truly incompressible while the gaseous phase follows a low Mach number approximation. A single field formulation is derived that can describe the whole flow field. The two phases are separated by a dynamic interface across which the physical properties of both phases are discontinuous, and on which surface tension forces are taken into account. Phase change however is not considered in the present article, and is the object of ongoing work. The numerical integration of the single field model is based on front tracking techniques that have been developed for purely incompressible flows in $[44,17,40]$.

The paper is organized as follows: section 2 is devoted to the physical modeling equations, considering the case of multiple gaseous inclusions in an incompressible liquid. A single field formulation of such multiphase flows is derived. In this section we also discuss some specific details concerning the link between the thermodynamic pressure in the bubbles and the pressure in the liquid phase. Numerical methods are presented in Section 3. A comparison with a single pressure method on an isentropic classical test case is provided in section 4, illustrating the high efficiency of our low Mach method. Section 5 presents numerical results for several non isothermal cases. A validation study performed on a reference 1D case is first presented. Next are investigated 2D cases consisting of air bubbles embedded in a closed cavity filled with liquid water. Conclusions and perspectives will finally be drawn in Section 6 .

\section{Physical modeling and governing equations}

\subsection{Specific models for each phase}

In this section we develop a single field formulation of a multiphase flow involving a strictly incompressible liquid phase and a compressible gaseous phase, the latter being considered under the low Mach number assumption. We con- 
sider a volume region of interest, $\Re$, containing a liquid phase (volume $\Omega_{l}$ ) and $\mathrm{N}$ gaseous inclusions. The volume of each bubble is $\Omega_{k}(t)(k=1,2, \ldots, N)$. No mass transfer occurs at the gas-liquid interfaces noted $\Sigma_{k}(t)$. Neglecting viscous loss in the energy balance, the following Navier-Stokes equations allow the description of the flow in the liquid and in the gas bubbles:

$$
\left\{\begin{array}{l}
\frac{\partial \rho}{\partial t}+\nabla \cdot(\rho \mathbf{v})=0 \\
\rho\left(\frac{\partial \mathbf{v}}{\partial t}+\mathbf{v} \cdot \nabla \mathbf{v}\right)=-\nabla p+\nabla \cdot \boldsymbol{\tau}+\rho \mathbf{g} \\
\rho c_{p}\left(\frac{\partial T}{\partial t}+\mathbf{v} \cdot \nabla T\right)=\nabla \cdot(k \nabla T)-\frac{T}{\rho}\left(\frac{\partial \rho}{\partial T}\right)_{p} \frac{D p}{D t} \\
p=f(\rho, T)
\end{array}\right.
$$

where $p(\mathbf{x}, t)$ is the pressure, $\rho$ the density, $\mathbf{v}$ the velocity, $T$ the temperature, $c_{p}$ the specific heat, $k$ the thermal conductivity and $\mathbf{g}$ the gravitational acceleration. The viscous tensor $\boldsymbol{\tau}$ is equal to $\lambda \nabla \cdot \mathbf{v I}+2 \mu \mathbf{D}$ with Lamé coefficients $\lambda$ and $\mu, \mathbf{D}$ being the strain rate tensor and $\mathbf{I}$ the identity tensor. The material derivative is denoted by $\frac{D}{D t}=\frac{\partial}{\partial t}+\mathbf{v} \cdot \nabla$.

The interfaces between the liquid and the gas inclusions act as surfaces of discontinuity and jump conditions have to be used $[8,12]$. As no mass transfer is considered in the present case, these conditions read:

$$
\left\{\begin{array}{l}
\mathbf{v}_{l} \cdot \mathbf{n}=\mathbf{v}_{g} \cdot \mathbf{n} \\
\left(p_{g}-p_{l}\right) \mathbf{I} \cdot \mathbf{n}=\left(\boldsymbol{\tau}_{g}-\boldsymbol{\tau}_{l}\right) \cdot \mathbf{n}+\sigma \kappa \mathbf{n} \\
\left(\mathbf{q}_{g}-\mathbf{q}_{l}\right) \cdot \mathbf{n}=0
\end{array}\right.
$$

where $\sigma$ is the surface tension coefficient, $\kappa$ is twice the mean interface curvature which is positive when the center of curvature lies in the gas, $\mathbf{n}$ is the unit normal to the interface, defined to point outside the gaseous phase and $\mathbf{q}$ is the heat flux. In (2) the subscripts $l$ and $g$ denote the values on the liquid and gaseous sides of the interface.

We will consider in the present paper two-phase systems where an incompressible liquid is in contact with various gas bubbles, each one of them having its own pressure. For the liquid phase of constant density $\rho_{l}$, the Navier Stokes equations (1) reduce to:

$$
\left\{\begin{array}{l}
\nabla \cdot \mathbf{v}=0 \\
\rho_{l}\left(\frac{\partial \mathbf{v}}{\partial t}+\mathbf{v} \cdot \nabla \mathbf{v}\right)=-\nabla p+\nabla \cdot \boldsymbol{\tau}+\rho_{l} \mathbf{g}(b) \\
\rho_{l} c_{p}\left(\frac{\partial T}{\partial t}+\mathbf{v} \cdot \nabla T\right)=\nabla \cdot(k \nabla T)
\end{array}\right.
$$

Now considering that bubbles are made of the same perfect gas, the Navier 
Stokes equations (1) read in the gaseous phase:

$$
\left\{\begin{array}{l}
\frac{\partial \rho}{\partial t}+\nabla \cdot(\rho \mathbf{v})=0 \\
\rho\left(\frac{\partial \mathbf{v}}{\partial t}+\mathbf{v} \cdot \nabla \mathbf{v}\right)=-\nabla p+\nabla \cdot \boldsymbol{\tau}+\rho \mathbf{g} \\
\frac{\partial T}{\partial t}+\mathbf{v} \cdot \nabla T=\frac{1}{\rho c_{p}} \nabla \cdot(k \nabla T)+\frac{\gamma-1}{\gamma} \frac{T}{p} \frac{D p}{D t} \\
p=\rho r T
\end{array}\right.
$$

where $r$ is the ideal gas constant $r=c_{p}-c_{v}$ and $\gamma=c_{p} / c_{v}$ is the ratio of specific heats.

The jump equations given by (2) are unchanged. The linear momentum jump condition notably shows that pressure is continuous through the interface provided that viscous and surface tension effects be negligible. Velocity should also be continuous at the interfaces by virtue of the continuity equation.

In this paper are solely considered gas liquid two phase flows and small velocities. In this particular case, the Mach number in the compressible gaseous phase is small. The flow in the bubbles may then be described using a low Mach model [33, 6, 24]. Such a model is derived from the fully compressible Navier Stokes equations (1) expanding each variable into a power series of the Mach number $M_{0}$, and taking the asymptotic limit for $M_{0}$ going to zero. For each variable, the lowest order term remains in the equations, except for the pressure $p(\mathbf{x}, t)$ which is split in two components, a thermodynamic pressure, uniform in space $P(t)$ and a hydrodynamic pressure $p_{2}(\mathbf{x}, t)$. One has $p(\mathbf{x}, t) \simeq P(t)+p_{2}(\mathbf{x}, t)$. As the ratio $p_{2}(\mathbf{x}, t) / P(t) \sim M_{0}^{2}$, the hydrodynamic pressure $p_{2}(\mathbf{x}, t)$ is much smaller than the thermodynamic pressure $P(t)$. For a single-phase compressible flow of a perfect gas, the low Mach model can be written, expressing the conservation of mass, momentum and energy (viscous loss is neglected in the energy balance):

$$
\left\{\begin{array}{l}
\frac{\partial \rho}{\partial t}+\nabla \cdot(\rho \mathbf{v})=0 \\
\rho\left(\frac{\partial \mathbf{v}}{\partial t}+\mathbf{v} \cdot \nabla \mathbf{v}\right)=-\nabla p_{2}+\nabla \cdot \boldsymbol{\tau}+\rho \mathbf{g} \\
\frac{\partial T}{\partial t}+\mathbf{v} \cdot \nabla T=\frac{1}{\rho c_{p}} \nabla \cdot(k \nabla T)+\frac{\gamma-1}{\gamma} \frac{T}{P} \frac{d P}{d t} \\
P=\rho r T
\end{array}\right.
$$

Using the ideal gas law $P(t)=\rho(\mathbf{x}, t) r T(\mathbf{x}, t)$, the continuity equation in (5) may also be written :

$$
\nabla \cdot \mathbf{v}=-\frac{1}{\rho} \frac{D \rho}{D t}=\frac{1}{T} \frac{D T}{D t}-\frac{1}{P} \frac{d P}{d t}
$$




\subsection{Derivation of a single field formulation}

We now have to build a single set of governing equations that can represent both phases. As our aim is to model the liquid phase as incompressible and the gas phase as compressible under the low Mach approximation, this set of equations must be consistent with equations (2), (3) and (5). In order to develop a generalized single field model, let us classically introduce a Heaviside function $H(\mathbf{x}, t)$, which is the characteristic function of the gaseous phase $(H$ is equal to 1 in the gas, and equal to 0 in the liquid phase). If $\mathrm{N}$ bubbles of volume $\Omega_{j}, j=1,2, \ldots N$ are present in $\Re$, each one is marked by its own characteristic function $H_{j}(\mathbf{x}, t)$ and its own thermodynamic pressure $P_{j}(t)$. The gas characteristic function $H(\mathbf{x}, t)$ is given by $H=\sum_{j=1}^{N} H_{j}$ since $\Omega_{j} \cap \Omega_{i}=\varnothing$ for $i \neq j$.

As no phase change is considered in the present paper, $H$ is simply advected by the flow, and thus obeys to the following transport equation (although this equation is not directly solved in the front tracking method that we use for interface treatment):

$$
\frac{\partial H}{\partial t}+\mathbf{v} \cdot \nabla H=0
$$

\subsubsection{Continuity and energy equations}

We also must define a generalized equation of state valid for the two phases. The liquid assumed to be incompressible has a constant density $\rho_{l}$. Using the perfect gas law, a two-phase generalized equation of state can be written as:

$$
\rho(\mathbf{x}, t)=\sum_{j=1}^{N} H_{j}(\mathbf{x}, t) \frac{P_{j}(t)}{r T(\mathbf{x}, t)}+(1-H(\mathbf{x}, t)) \rho_{l}
$$

Using (6), (7) and (8), it is now possible to establish a generalized continuity equation valid in both liquid $(H=0)$ and gas $(H=1)$ phases :

$$
\nabla \cdot \mathbf{v}=H(\mathbf{x}, t) \frac{1}{T} \frac{D T}{D t}-\sum_{j=1}^{N} H_{j}(\mathbf{x}, t) \frac{1}{P_{j}} \frac{d P_{j}}{d t}
$$

Similarly is established a generalized energy equation:

$$
\left(\frac{\partial T}{\partial t}+\mathbf{v} \cdot \nabla T\right)=\frac{1}{\rho c_{p}} \nabla \cdot(k \nabla T)+\frac{\gamma-1}{\gamma} T \sum_{j=1}^{N} H_{j}(\mathbf{x}, t) \frac{1}{P_{j}} \frac{d P_{j}}{d t}
$$


The thermodynamic pressure may be calculated using an integral relation that is now derived. Let us suppose that the gaseous phase $\Omega_{j}(t)$ is enclosed by walls or by the liquid phase (case of a bubble for example), and let us denote by $\mathbf{n}$ the unit outward normal to the bounding surface $\Sigma_{j}(t)$. We integrate the mass equation (9) over $\Omega_{j}(t)$ to obtain:

$$
\frac{1}{P_{j}} \frac{d P_{j}}{d t}=\frac{1}{\int_{\Omega_{j}(t)} d \mathbf{x}}\left(\int_{\Omega_{j}(t)} \frac{1}{T} \frac{D T}{D t} d \mathbf{x}-\int_{\Omega_{j}(t)} \nabla \cdot \mathbf{v} d \mathbf{x}\right)
$$

or equivalently:

$$
\frac{1}{P_{j}} \frac{d P_{j}}{d t}=\frac{1}{\int_{\Re} H_{j} d \mathbf{x}}\left(\int_{\Re} H_{j} \frac{1}{T} \frac{D T}{D t} d \mathbf{x}-\int_{\Sigma_{j}(t)} \mathbf{v} \cdot \mathbf{n} d s\right)
$$

This formulation allows for the calculation of the source term $\frac{1}{P_{j}} \frac{d P_{j}}{d t}$ in the energy equation (10). Once $\frac{1}{P_{j}} \frac{d P_{j}}{d t}$ is known, the pressure $P_{j}(t)$ can be obtained directly from a time integration, i.e.:

$$
P_{j}(t)=P_{j}\left(t_{0}\right) \cdot \exp \int_{t_{0}}^{t}\left(\frac{1}{P_{j}} \frac{d P_{j}}{d t^{\prime}}\right) d t^{\prime}
$$

Let us note for further usage that, owing to incompressibility, the surface term integration in (12) can be done over any closed contour in the liquid enclosing the gaseous zone $\Omega_{j}(t)$.

An important point to emphasize concerning the above model is that, in addition to the increased accuracy that can be expected from the splitting of the pressure into two components of different magnitudes, the calculation of the thermodynamic pressure using the integral relation (11) constitutes a means of imposing mass conservation of the gaseous phase separately. This is a very important advantage over other methods based on the use of a single pressure field, as will be illustrated on 1D numerical results (section 4).

\subsubsection{Momentum equation and pressure issues}

We now have to consider the momentum equation issue. Its treatment cannot be as straightforward as for continuity and energy equations and deserves a few preliminary comments. Even though the momentum equations as written in (3-b) and (5-b) appear to be very similar, an essential difference arises due to the pressure gradient term. Let us recall that the problems of interest in the present paper concern a liquid volume with several gaseous inclusions, each one having its own (thermodynamic) pressure. The linear momentum jump equation (2-b) shows that for any interface, the local pressure is the same on both gaseous and liquid sides (viscous and surface tension effects are disregarded here for the sake of comprehension). The difference in the bubbles' pressure therefore generates a pressure gradient in the liquid volume 
which induces in turn an acceleration of the liquid. This pressure gradient, $\nabla p$ in Eq. (3-b), is expected to scale as $P_{0} / L_{0}$ where $L_{0}$ and $P_{0}$ are respectively the characteristic values for the liquid dimension and for the thermodynamic pressure in the bubbles. On the other hand, the pressure $p_{2}$ defined in the low Mach model (5) scales as $M_{0}^{2} P_{0}$ leading the pressure gradient in Eq. (5-b) to scale as $M_{0}^{2} P_{0} / L_{0}$. At that point, it is obvious that a proper description of the incompressible liquid flow cannot be achieved through momentum equation (5b). It is clear however that the problem does not arise if the thermodynamic pressure, although being constant, is retained in the momentum equation of the low Mach model (5-b), that can equivalently be written:

$$
\rho\left(\frac{\partial \mathbf{v}}{\partial t}+\mathbf{v} \cdot \nabla \mathbf{v}\right)=-\nabla\left(p_{2}+P\right)+\nabla \cdot \boldsymbol{\tau}+\rho \mathbf{g}
$$

When using equation (14) to derive the momentum jump equation at the interfaces, which now reads:

$$
\left(\left(p_{2}+P\right)_{g}-p_{l}\right) \mathbf{I} \cdot \mathbf{n}=\left(\boldsymbol{\tau}_{g}-\boldsymbol{\tau}_{l}\right) \cdot \mathbf{n}+\sigma \kappa \mathbf{n},
$$

then the total pressure at the gas side has the proper magnitude.

The splitting of pressure in the gas suggests a corresponding splitting of pressure in the liquid, aimed at providing a single momentum equation valid for both phases. We thus propose to split the liquid pressure field into two components $P_{l}$ and $\pi_{l}$ that is:

$$
p(\mathbf{x}, t)=\pi_{l}(\mathbf{x}, t)+P_{l}(\mathbf{x}, t)
$$

in the liquid.

We choose to define the respective components of the pressure in such a way that the momentum jump relations split like:

$$
\left\{\begin{array}{l}
\left(p_{2 g}-\pi_{l}\right) \mathbf{I} \cdot \mathbf{n}=\left(\boldsymbol{\tau}_{g}-\boldsymbol{\tau}_{l}\right) \cdot \mathbf{n}+\sigma \kappa \mathbf{n}(a) \\
\left(P_{g}-P_{l}\right) \mathbf{I} \cdot \mathbf{n}=0
\end{array}\right.
$$

i.e. the jump relations due to surface tension and viscous tensor are ascribed to $p_{2 g}$ and $\pi_{l}$ whereas $P_{g}$ and $P_{l}$ are equal at the liquid gas interface.

In addition, in the same way as $p_{2}$ is the Lagrangian multiplier of the continuity equation in the gas, we ascribe to $\pi_{l}$ its respective role of Lagrangian multiplier of the divergence free continuity equation in the liquid. This will later be used to solve simultaneously for $p_{2}$ and $\pi_{l}$ in the framework of a single field equation in the projection step of the time stepping algorithm. This is achieved by requiring that $P_{l}$ be harmonic in the liquid since the divergence of its gradient is then identically zero.

Finally let us call $P_{e}(\mathbf{x}, t)$ (extended thermodynamic pressure field) the pressure field that is equal to $P_{j}(t)$ in each bubble and to $P_{l}(\mathbf{x}, t)$ in the liquid. 
$P_{e}$ is continuous at the interfaces from (17-b). Similarly we call $\pi$ the hydrodynamic pressure field that is equal to $p_{2}$ in the gas, and to $\pi_{l}$ in the liquid. We thus have built two pressure fields defined in the whole domain, their sum giving the correct jump relations at the interfaces. Using these two pressure fields a single field momentum equation capable of treating both phases can be written as:

$$
\rho\left(\frac{\partial \mathbf{v}}{\partial t}+\mathbf{v} \cdot \nabla \mathbf{v}\right)=-\nabla \pi-\nabla P_{e}+\nabla \cdot \boldsymbol{\tau}+\rho \mathbf{g}
$$

Let us now construct the equation for this auxiliary field $P_{e}(\mathbf{x}, t)$. As said above, $P_{e}$ satisfies:

$$
\begin{cases}\nabla^{2} P_{e}(\mathbf{x}, t)=0, & \text { in the liquid } \\ P_{e}(\mathbf{x}, t)=P_{j}(t), & \text { in each gas bubble }\end{cases}
$$

Moreover, since this auxiliary pressure field should not modify the velocity at the domain boundaries, $P_{e}(\mathbf{x}, t)$ satisfies an homogeneous Neumann boundary condition on the walls of any closed domain. The above constraints are summarized in the following equation for $P_{e}$ :

$$
\frac{1}{\eta^{2}} P_{e}(\mathbf{x}, t) \cdot H(\mathbf{x}, t)+(1-H(\mathbf{x}, t)) \cdot \nabla^{2} P_{e}(\mathbf{x}, t)=\sum_{j=1}^{N} H_{j}(\mathbf{x}, t) \cdot \frac{1}{\eta^{2}} P_{j}(t)
$$

supplemented with homogeneous Neumann boundary conditions on the walls. The quantity $\eta$, which is homogeneous to a length, is introduced in the composite equation (20) for dimensional consistency. If the Heaviside function $H$ was strictly a characteristic function equal to either 0 or 1 , the value of $\eta$ would have no influence on the solution. However, in the numerical implementation of the model, $H$ is smoothed over a few mesh cells, leading to the existence of a transition zone (of fixed width) between the two pure phases where $H$ can take values between 0 and 1 (see section 3.1). The influence of $\eta$ on the numerical solution is analyzed in section 3.2.

As a summary, the governing equations of the single field model are now:

$$
\left\{\begin{array}{l}
\nabla \cdot \mathbf{v}=H(\mathbf{x}, t) \frac{1}{T} \frac{D T}{D t}-\sum_{j=1}^{N} H_{j}(\mathbf{x}, t) \frac{1}{P_{j}} \frac{d P_{j}}{d t} \\
\frac{D \mathbf{v}}{D t}=-\frac{1}{\rho} \nabla \pi-\frac{1}{\rho} \nabla P_{e}+\frac{1}{\rho} \nabla \cdot \boldsymbol{\tau}+\mathbf{g} \\
\frac{D T}{D t}=\frac{1}{\rho c_{p}} \nabla \cdot(k \nabla T)+\frac{\gamma-1}{\gamma} T \sum_{j=1}^{N} H_{j}(\mathbf{x}, t) \frac{1}{P_{j}} \frac{d P_{j}}{d t}
\end{array}\right.
$$


Surface tension was not included so far. It can be taken into account by adding in the right hand side of the momentum equation (21-b) the following integral source term acting on the interface:

$$
-\frac{1}{\rho} \int_{\Sigma(t)} \sigma \kappa \mathbf{n} \delta\left(\mathbf{x}-\mathbf{x}_{s}\right) d s
$$

where $\mathbf{x}_{s}=\mathbf{x}(s, t)$ is a parametrization of $\Sigma(t)$. The function $\delta\left(\mathbf{x}-\mathbf{x}_{s}\right)$ is a three-dimensional delta function that is non-zero only where $\mathbf{x}=\mathbf{x}_{s}$. We also use $H$ to construct the material property fields of the fluid for both the liquid and gaseous phases. For example, one way to construct the viscosity field is given by:

$$
\mu(\mathbf{x}, t)=\mu_{l}+\left(\mu_{g}-\mu_{l}\right) H(\mathbf{x}, t)
$$

where the subscripts $g$ and $l$ refer to the gas and liquid phases respectively. Similar equations can be written for the thermal conductivity, $k$, constant volume, $c_{v}$, or constant pressure, $c_{p}$, specific heats.

The jump conditions associated to system (21) are given by Eqs. (2-a), (2-c), (17). These conditions are not explicitly needed for our formulation, and are not used in the numerical method. However we will check that they are indeed satisfied by the numerical solution.

\subsection{Illustration on a 1-D Cartesian case}

As an illustration, we apply this model on a simple 1-D Cartesian case consisting of a moving liquid zone $\Omega_{l}=\left[x_{1}, x_{2}\right]$ of constant length $L=x_{2}-x_{1}$, enclosed between two gaseous zones $\Omega_{1}$ and $\Omega_{2}$ of variable length. This case was investigated in detail in [10], using an ALE approach where liquid and gaseous domains were considered separately. The thermodynamic pressures in the gaseous zones are $P_{1}(t)$ and $P_{2}(t)$. We consider here a simplified problem with no gravity and no viscous effects. Following (3), the formulation of the dynamic problem in the liquid is:

$$
\left\{\begin{array}{l}
\frac{\partial u_{l}}{\partial x}=0 \\
\frac{\partial u_{l}}{\partial t}+u_{l} \frac{\partial u_{l}}{\partial x}=-\frac{1}{\rho_{l}} \frac{\partial p_{l}}{\partial x}
\end{array}\right.
$$

The continuity equation shows that $u_{l}(x, t)=u_{l}(t)$. The pressure gradient is thus uniform in the liquid and may be written :

$$
\frac{\partial p_{l}}{\partial x}=\frac{p_{l}\left(x_{2}, t\right)-p_{l}\left(x_{1}, t\right)}{L}
$$

In 1-D Cartesian co-ordinates, the momentum jump equation (2-b) reduces to:

$$
p_{g}\left(x_{k}, t\right)=p_{l}\left(x_{k}, t\right) \quad k=1,2
$$


Using a low Mach model for the gas description, the pressure on the gaseous side of any interface reads:

$$
p_{g}\left(x_{k}, t\right)=P_{k}(t)+\pi_{g}\left(x_{k}, t\right) \quad k=1,2
$$

Splitting the pressure $p_{l}$ in two components $P_{e}$ and $\pi_{l}$, it comes:

$$
\frac{\partial p_{l}}{\partial x}=\frac{\partial P_{e}}{\partial x}+\frac{\partial \pi_{l}}{\partial x}
$$

Both terms in the r.h.s. are next identified using Eqs (25-28):

$$
\begin{aligned}
& \frac{\partial P_{e}}{\partial x}=\frac{P_{2}(t)-P_{1}(t)}{L} \\
& \frac{\partial \pi_{l}}{\partial x}=\frac{\pi_{l}\left(x_{2}, t\right)-\pi_{l}\left(x_{1}, t\right)}{L}
\end{aligned}
$$

In this particular 1-D Cartesian case, both pressure fields $P_{e}$ and $\pi$ are linear in the liquid. Using Eqs (24)-(27), the momentum equation in the liquid may also be written:

$$
\begin{gathered}
\rho_{l} L \frac{d u_{l}}{d t}=\left[P_{1}(t)+\pi_{g}\left(x_{1}, t\right)\right]-\left[P_{2}(t)+\pi_{g}\left(x_{2}, t\right)\right] \\
\rho_{l} L \frac{d u_{l}}{d t} \cong P_{1}(t)-P_{2}(t)
\end{gathered}
$$

One recognizes here the second law of Newton applied to the whole liquid domain $\Omega_{l}$. It states that a liquid acceleration arises from the difference between the (thermodynamic) pressure forces in the two gaseous media.

\section{$3 \quad$ Numerical method}

\subsection{Interface treatment}

The model described above must be coupled to a specific method for tracking the liquid-gas interface. Among the existing methods, we have chosen here to use the Lagrangian front tracking method developped in [11], [17], [44] and subsequent papers. A robust and connectivity free method to reconstruct the interface for both two and three-dimensional flows is discussed in [40]. This method uses a Lagrangian discretization and movement of the interface. The interface is represented by separate, non-stationary computational elements (straight lines in $2 \mathrm{D}$, triangles in 3D) which are physically connected at their vertices to form a Lagrangian interface mesh which lies within a stationary Eulerian finite-difference mesh. The interface elements are used to calculate 
geometric information and their vertices are advected in an explicit Lagrangian way, by integrating :

$$
\frac{d \mathbf{x}_{s}}{d t}=\mathbf{v}
$$

A Heaviside function $H(\mathbf{x}, t)$, defined on the Eulerian grid, is found from the Lagrangian definition of the interface at each time step, by solving the following Poisson equation:

$$
\nabla^{2} H=\nabla \cdot \int_{\Sigma(t)} \mathbf{n} \delta\left(\mathbf{x}-\mathbf{x}_{s}\right) d s
$$

using the definitions introduced in section 2. Once $H(\mathbf{x}, t)$ has been determined from $\mathbf{x}_{s}$ we can use it to construct values for the material property fields of the fluid for both the liquid and gaseous phases, using Eq. (23) and similar equations for $k$ and $c_{p}$.

At each time step, information must be passed between the moving Lagrangian interface and the stationary Eulerian grid since the Lagrangian interface points do not necessarily coincide with the Eulerian grid points. This is done by a method that has become known as the Immersed Boundary Technique which was introduced by Peskin [34] for the analysis of blood flow in the heart. With this technique, the infinitely thin interface is approximated by a smooth distribution function that is used to distribute the forces at the interface over grid points nearest the interface. In a similar manner, this function is used to interpolate field variables from the stationary grid to the interface. In this way, the front is given a finite thickness on the order of the mesh size to provide stability and smoothness. This leads to the existence of a "mixing" zone between the two fluids, where the model has no real physical meaning. Although the interface is smoothed, the method however is free from numerical diffusion since the interface thickness remains constant for all time, as it is directly controlled through the definition of the smoothed delta function, which is built on the Lagrangian definition of the interface. The question of the convergence of the method was considered, for example, in [11], [44]. Surface tension is treated using the method developed in [39], which was shown to minimize the parasitic currents that are commonly generated by inadequate numerical representation of surface tension.

\subsection{Fixing the parameter $\eta$ in the equation for extended pressure}

In this section we study the behavior of the function $P_{e}$ in the transition zone that is created at the interfaces by the Immersed Boundary technique. Without loss of generality, we restrict our study to the one-dimensional case for clarity reasons. Let us consider an isolated transition zone of width $l$ between gas at the left, at thermodynamic pressure $P_{0}(t)$, and liquid at the right. Let $y=x / l$ be the non dimensional abscissa describing the zone $(0 \leq y \leq 1)$ 
( $x=0$ corresponding to the left boundary of the transition zone). Setting $\chi(y)=H(x, t)$ and $f(y)=P_{e}(y, t)-P_{0}(t)$, equation (20) reads:

$$
\chi(y) f(y)+(1-\chi(y)) \frac{\eta^{2}}{l^{2}} f^{\prime \prime}=0
$$

The boundary conditions associated to (34) are : $f(0)=0, f^{\prime}(1)=l g_{P}$, where $g_{P}$ is the thermodynamic pressure gradient across the liquid imposed by the existence of another separated gaseous area (the extended pressure profile being harmonic is linear in the liquid in the 1D case). Although Eq. (34) can be solved only for simple $\chi$ profiles, it is interesting to study its behavior for extreme values of the parameter $\epsilon=\eta / l$. For $\epsilon \ll 1$, we get $f(y) \approx 0$, that is $P_{e}(x, t) \approx P_{0}(t)$ in the transition zone. For $\epsilon \gg 1$ we get $f(y) \approx l_{g_{P}} y$, that is $P_{e}(x, t) \approx P_{0}(t)+g_{P} x$. This leads to conclude that the difference between the two values of $\epsilon$ results in a shift of the global $P_{e}$ profile by the length $l$, the linear part starting at the gaseous side in the case $\epsilon \gg 1$ and at the liquid side in the case $\epsilon \ll 1$. In the case where $\epsilon$ takes intermediate values, for instance $\epsilon=1$, the profile of $P_{e}$ will result in an intermediate curve, with the same gradient at the liquid side (see Fig. 1 left).

The question is now to analyze whether what happens in the transition zone has some influence on the gradient of the calculated field $P_{e}$. To this end we must take into account a second gaseous zone that we suppose at thermodynamic pressure $P_{1}$, which will fix the extended pressure gradient inside the liquid. Considering the previous analysis, we can conclude that, in function of the value of $\epsilon$, the linear variation of the calculated $P_{e}$ takes place between the two gaseous sides $(\epsilon \gg 1)$, or between the two liquid sides $(\epsilon \ll 1)$. This will change the extended pressure gradient of an amount proportional to the width of the transition zone (thus on the order of the mesh size) as is illustrated in Fig. 1 (right). The exact value of the gradient will be obtained if the linear part of $P_{e}$ originates at the interface, that is in the middle of the transition zone. Indeed the error will be reduced with mesh refinement. The error also decreases when the ratio of the transition zone length to liquid width is small.

From the previous analysis we can conclude that $\eta$ should be of the same order than $l$, although any other value will give the correct result at grid convergence. In results presented in the following, we fixed $\eta=l=4 \delta x, \delta x$ being the grid cell size.

\subsection{Discretization and Projection method}

The spatial discretization of model (21)-(11)-(20) is based on centered finite differences for both the convection and diffusion terms. A staggered mesh is used, where density, hydrodynamic pressure and temperature are located at the center of the cells, while the components of the velocity are located on the 
faces. We have used here a first order explicit temporal discretization, but the extension to semi-implicit second order should be considered in the future. A classical prediction-projection algorithm is used to compute the velocities [15]. The predicted velocity $\mathbf{v}^{*}$ is calculated from:

$$
\frac{\mathbf{v}^{*}-\mathbf{v}^{n}}{\delta t}=-\mathbf{v}^{n} \cdot \nabla \mathbf{v}^{n}+\frac{1}{\rho^{n+1}} \nabla \cdot \boldsymbol{\tau}^{n}-\frac{1}{\rho^{n+1}} \nabla \pi^{n}-\frac{1}{\rho^{n+1}} \nabla P_{e}^{n}+\mathbf{g}
$$

Calculating $\mathbf{v}^{*}$ from equation (35) needs to calculate first the extended thermodynamic pressure field $P_{e}$. To this end Eq. (20) is solved using a BiCGStab solver.

Once $\mathbf{v}^{*}$ is known, the projection step consists in extracting $\mathbf{v}^{n+1}$ from $\mathbf{v}^{*}$ in such a way that $\nabla \cdot \mathbf{v}^{n+1}$ is zero in the liquid, and equal to its specified value in the gas. This amounts to solving the following Poisson equation for the hydrodynamic pressure:

$$
\nabla \cdot\left(\frac{1}{\rho^{n+1}} \nabla \phi\right)=\frac{1}{\delta t}\left(\nabla \cdot \mathbf{v}^{*}-\nabla \cdot \mathbf{v}^{n+1}\right)
$$

where $\phi=\pi^{n+1}-\pi^{n}$ is the pressure increment. In the r.h.s. of equation (36), $\nabla \cdot \mathbf{v}^{n+1}$ is obtained using the mass conservation equation (21-a), after the temperature field and $\frac{1}{P_{j}} \frac{d P_{j}}{d t}$ in each gaseous inclusion have been calculated. The latter are calculated using a more convenient form of Eq. (11):

$$
\frac{1}{P_{j}} \frac{d P_{j}}{d t}=\frac{1}{\int_{\Re} H_{j} d \mathbf{x}}\left(\int_{\Re} H_{j} \frac{1}{T} \frac{D T}{D t} d \mathbf{x}-\int_{\Re} H_{j} \nabla \cdot \mathbf{v} d \mathbf{x}\right)
$$

The solution of (36) requires a robust matrix solver as the coefficients $1 / \rho$ are discontinuous, the ratio between the gas and liquid density being generally very large (around 1000 for air-water, much more for example if the liquid is molten glass). To this end, we have used a multigrid method that is described in Appendix A.

Once the hydrodynamic pressure is obtained, the velocity is calculated from:

$$
\mathbf{v}^{n+1}=\mathbf{v}^{*}-\delta t \frac{1}{\rho^{n+1}} \nabla \phi
$$

and the hydrodynamic pressure field is updated by:

$$
\pi^{n+1}=\pi^{n}+\phi
$$

Let us now sum up the complete numerical methodology for the determination of the solution at time $t=(n+1) \delta t$, assuming the solution known at time $t=n \delta t$. Due to the non linear coupling of the equations, iterations are necessary within each time step. Indeed, the calculation of $\left(\frac{1}{P} \frac{d P}{d t}\right)_{j}^{n+1}$ using Eq.(37) makes use of the velocity $\mathbf{v}^{n+1}$ which is unknown at the first iteration. 
A convenient first guess for $\mathbf{v}^{n+1}$ can be obtained from the discretization of a simplified momentum equation, where only the external forces (extended thermodynamic and gravity forces) are taken into account. It is important to include the extended pressure force in the first guess in order to avoid selecting the trivial solution consisting of fluid at rest. For this solution, in the absence of gravity, the hydrodynamic pressure field is obtained as the opposite of the thermodynamic pressure field.

The whole procedure is then:

(1) using the front-tracking method, calculate $H_{j}^{n+1}$ in each gaseous zone, $H^{n+1}=\sum_{j=1}^{N} H_{j}^{n+1}$ and the new values of $k, c_{p}$

(2) Set guessed estimates $P_{j}^{n+1}=P_{j}^{n},\left(\frac{1}{P} \frac{d P}{d t}\right)_{j}^{n+1}=\left(\frac{1}{P} \frac{d P}{d t}\right)_{j}^{n}, \rho^{n+1}=\rho^{n}$, $\mathbf{v}^{n+1}=\mathbf{v}^{n}-\delta t \frac{1}{\rho^{n}} \nabla P_{e}^{n}+\delta t \mathbf{g}$

(3) • solve Eq. (21-c) for $T^{n+1}$ using $\left(\frac{1}{P} \frac{d P}{d t}\right)_{j}^{n+1}$

- calculate the new value of $\left(\frac{1}{P} \frac{d P}{d t}\right)_{j}^{n+1}$ and $P_{j}^{n+1}$ in each gaseous zone using $\mathbf{v}^{n+1}, H^{n+1}$ and $T^{n+1}$ in (37) and (13)

- calculate $\rho^{n+1}$ using (8)

- calculate the extended thermodynamic pressure $P_{e}^{n+1}(\mathbf{x}, t)$ from $(20)$ using $P_{j}^{n+1}, H_{j}^{n+1}$

- calculate $\nabla \cdot \mathbf{v}^{n+1}$ for the Poisson equation using the mass equation (21-a)

- calculate the predicted velocities $\mathbf{v}^{*}$ from (35)

- solve (36) for the hydrodynamic pressure increment $\phi$ (multigrid)

- project the velocity by (38)

- increment the hydrodynamic pressure by (39)

(4) If the computed thermodynamical pressure, density and velocity are different from the ones used at the beginning of step 3, restart from step 3 for a new iteration.

In the computations presented in this study, two to four iterations were needed for the convergence of the thermodynamical pressure. However the results with or without internal iterations were indistinguishable. This might not be true for other types of flows.

For closed gaseous zones, an important point to emphasize is the compatibility relation that must be satisfied when processing the integration of (37) to calculate $\left(\frac{1}{P} \frac{d P}{d t}\right)^{n+1}$ in each gaseous zone. In the case where the fluid domain is bounded by walls and includes $N$ closed gaseous zones, replacing the $N$ equations (37) in (21-a) and integrating over the whole domain results in the following relation:

$$
\sum_{j=1}^{N} \int_{\Omega_{j}(t)} \nabla \cdot \mathbf{v} d \mathbf{x}=0
$$


However, in the front-tracking method, as already mentioned, there exists a mixing zone, a few cells thick, inside which the velocity results from the blending of the liquid velocity, which is divergence free, and the gas velocity which is not. Thus there is no chance that the global balances (40) be zero if the contour of integration for each gaseous zone is located inside this mixing zone. In fact the contour of integration must include the mixing zone up to the liquid, or equivalently the volume integral in (40) must take into account all grid cells for which $H_{j}$ is not zero. To do this we define an "enlarged" function $H_{j}^{e x t}$, such that:

$$
\begin{cases}H_{j}^{e x t}(\mathbf{x}, t)=1, & \text { if } \quad H_{j}(\mathbf{x}, t) \neq 0 \\ H_{j}^{e x t}(\mathbf{x}, t)=0, & \text { if } \quad H_{j}(\mathbf{x}, t)=0\end{cases}
$$

This enlarged characteristic function is used for the integration of (37) that becomes :

$$
\frac{1}{P_{j}} \frac{d P_{j}}{d t}=\frac{1}{\int_{\Re} H_{j} d \mathbf{x}}\left(\int_{\Re} H_{j} \frac{1}{T} \frac{D T}{D t} d \mathbf{x}-\int_{\Re} H_{j}^{e x t} \nabla \cdot \mathbf{v} d \mathbf{x}\right)
$$

\section{Comparison of the 1D low Mach method with a single pressure method on the Oscillating Water Column test case}

In this section we highlight the capability of our low Mach method to produce accurate results, even when the time step is of the order of the convective time step, i.e. large compared to an explicit acoustic stability criteria. On the opposite, we show that a method involving a single pressure (falling in the class of all Mach methods) needs to be used with small time steps, as it produces very large numerical diffusion when too large time steps are used. It is likely that other single pressure methods, which use more or less the same equation for the pressure, will lead to similar conclusions. This demonstrates that a very significant gain in efficiency is obtained using our low Mach method. Moreover the gain is expected to be inversely proportional to the Mach number.

We consider the oscillating water column, a 1D isentropic problem that is treated in [19] and [18]. The problem is as follows: a closed 1D tube is filled with a column of water in between of two columns of air. We use the same physical settings as in [19],[18] (see Fig. 2). At initial state, $x_{f s}=0.1$ and all three columns flow to the right at constant speed $u_{0}=1$ and constant pressure $p_{0}=1$. Starting from initial state, the air to the right is compressed by the water while the air to the left expands, generating a pressure difference across the water column. The water column then starts to oscillate. In the following 
results we use the isentropic equation of state for air:

$$
p / p_{0}=\left(\rho / \rho_{0}\right)^{1 / \gamma}
$$

with $\gamma=1.4$, and initial value $\rho_{0}=0.001$. This also sets the reference speed of sound value in air $c_{a}=\left(\gamma p_{0} / \rho_{0}\right)^{1 / 2}=37.42$. Water is considered as incompressible, of constant density $\rho_{w}=1$, and the fluid viscosities are set to zero. In our low Mach model, the thermodynamic pressure $P$ is used in (43). Let us notice that in this problem the Mach number in the gas never exceeds 0.03 as we have verified, justifying the low Mach assumption.

In [19], water and air are both treated as compressible fluids, and an explicit method is used. The work in [18] also treats the water as compressible, and makes use of a single pressure implicit method aimed at solving all speed multi-phase flows, where a Helmholtz equation comparable to the one in [7] is solved for pressure. However in [18] the gas-dynamics equations are not solved in the gas, avoiding the difficulties of a real two-phase flow computation.

Let us briefly describe the single pressure method that we use for these computations. The flow model is a single pressure isentropic version of (21), that writes:

$$
\left\{\begin{array}{l}
\nabla \cdot \mathbf{v}=-H(\mathbf{x}, t) \frac{1}{\rho} \frac{D \rho}{D t}=-H(\mathbf{x}, t) \frac{1}{\gamma p} \frac{D p}{D t} \\
\frac{\partial \mathbf{v}}{\partial t}+\mathbf{v} \cdot \nabla \mathbf{v}+\frac{1}{\rho} \nabla p=\frac{1}{\rho} \nabla \cdot \boldsymbol{\tau}+\mathbf{g}
\end{array}\right.
$$

where $p$ is the (unique) pressure that is involved in the equation of state. We first approximate the continuity equation by:

$$
\nabla \cdot \mathbf{v}^{n+1}=-H^{n+1} \frac{1}{\gamma p^{*}}\left(\frac{p^{n+1}-p^{n}}{\delta t}+\mathbf{v}^{*} \nabla p^{n+1}\right)
$$

$\mathbf{v}^{*}$ being the predicted velocity and $p^{*}$ obtained by second order backward time extrapolation. This results in the following equation for the pressure:

$$
\nabla \cdot\left(\frac{1}{\rho^{*}} \nabla \phi\right)-\frac{1}{\delta t^{2}} \frac{H^{n+1}}{\gamma p^{*}} \phi-\frac{1}{\delta t} \frac{H^{n+1}}{\gamma p^{*}} \mathbf{v}^{*} \nabla \phi=\frac{1}{\delta t} \nabla \cdot \mathbf{v}^{*}+\frac{1}{\delta t} \frac{H^{n+1}}{\gamma p^{*}} \mathbf{v}^{*} \nabla p^{n}
$$

with $\rho^{*}=H^{n+1} \rho_{0}\left(p^{*} / p_{0}\right)^{\gamma}+\left(1-H^{n+1}\right) \rho_{w}$.

We first present the results obtained using our two-pressure low Mach method. For comparison with an explicit method and the results in [19] and [18], the time step is calculated as a multiple of the one obtained by using the stability criteria in [19]:

$$
\delta t=C F L \frac{\delta x}{\max _{j}\left|u_{j}\right|+c_{w}}
$$

where $c_{w}$ is the value of speed of sound in water used in [19], $c_{w}=144.94$. In the explicit case the stability criterion is $C F L \leq 1$, while a stability criterion based on the convective velocity would allow values of $C F L$ of the order of 100 . 
We present results for a uniform grid with $\delta x=1 / 40$. Fig. 3 presents the time evolution of the pressure coefficients obtained using our low Mach model, for two values of the $C F L$ number, $C F L=1$ and $C F L=100$. The pressure coefficients are defined as

$$
P(t)=\frac{p\left(x_{w a l l}, t\right)-p_{0}}{p_{0}}
$$

where $x_{\text {wall }}$ denote the positions at the left and right walls. The pressure $p$ in (48) is taken as the thermodynamic pressure for the low Mach method. One can remark that the curves corresponding to $C F L=1$ and $C F L=100$ are nearly superimposed, indicating that the method is accurate even using large time steps. Surprisingly, the pressure levels are the same as in [18], which are different from those in [19], although we use a two-fluid model. On the contrary, corresponding results using the single pressure method, presented in Fig. 4, exhibit a very large dependence on the time step. For $C F L=1$, the results coincide with those given by the low Mach method. For $C F L=100$, the oscillation of the flow decays due to significant numerical dissipation. This implies that the single pressure method can be used only with small values of the $C F L$ number based on acoustic speed, leading to poor efficiency.

Fig. 5 and Fig. 6 present the time evolution of the relative error of total mass of air in the tube for the two methods. The error presents a similar amplitude, about $10^{-3}$, for the two methods at $C F L=1$. This value is close to the one reported in [19]. It is much higher than the mass conservation error reported in $[18]\left(10^{-7}\right)$. However in this work the interface was considered as a moving boundary, described using a level set method combined with a ghost fluid treatment that avoids the errors due to the jump of the fluids properties. For $C F L=100$, the error of the low Mach method is only about 3-4 times the error for $C F L=1$, which shows that the gain in efficiency is not accompanied by an error increase in the same proportions. On the contrary, the single pressure method generates much larger mass conservation errors for large $C F L$ values. Moreover the total mass of air is decreasing, meaning that air is converted into water. This phenomenon, that was also observed in [19], does not appear when using the low Mach method, the mass of each fluid being conserved separately.

We conclude this section with a grid convergence study of the low Mach method for $C F L=100$. Fig. 7 presents the velocity fields at time $t=10$, obtained for increasing grid resolution : $\delta x=1 / 40, \delta x=1 / 80, \delta x=1 / 160$, $\delta x=1 / 320$. In the liquid column the velocity is uniform due to incompressibility, while it is linear in the gaseous columns. One can notice that the differences between the four grids are small, and that convergence is nearly reached for $\delta x=1 / 80$. Moreover the velocity appears continuous at the interfaces, showing that the jump relations are indeed satisfied. 


\section{Investigations of Air-Water configurations using the low Mach method}

All the simulations presented below involve water and air as the incompressible and compressible fluid respectively. Surface tension is taken into account in the $2 \mathrm{D}$ case, except for case 1 . For water, the physical characteristics are the following : density $\rho_{l}=1000 \mathrm{~kg} / \mathrm{m}^{3}$, dynamic viscosity $\mu_{l}=0.001$ Pa.s, thermal conductivity $k_{l}=0.6 \mathrm{Wm}^{-1} \mathrm{~K}^{-1}$, specific heat $c_{p l}=4184 \mathrm{JK}^{-1} \mathrm{~kg}^{-1}$. For air, considered as a perfect gas, the values are : dynamic viscosity $\mu_{g}=$ $1.8210^{-5}$ Pa.s, thermal conductivity $k_{l}=0.0256 \mathrm{Wm}^{-1} \mathrm{~K}^{-1}$, specific heat at constant pressure $c_{p g}=1004.5 \mathrm{JK}^{-1} \mathrm{~kg}^{-1}$, specific heat ratio $\gamma=1.4$. For air/water interfaces the surface tension coefficient is $\sigma=0.07 \mathrm{Nm}^{-1}$.

The time step that is used in these simulations is restricted by the explicit stability condition imposed by the diffusive part of the equations: viscous and thermal conduction effects, the latest being generally the most restrictive. Thus the time step is taken as $\delta t=0.25 \delta x^{2} / \max \left(k / \rho c_{p}\right)$. Using an implicit scheme for the diffusive terms of the Navier Stokes equations would remove this restriction and allow for the use of a convective time step.

\subsection{Numerical results for a $1 D$ non-isothermal problem}

A one-dimensional fluid system consisting of two layers of gas (air) enclosing a layer of liquid (water) is considered. The system is closed by two walls. The total length of the system is $100 \mu \mathrm{m}$, the length of the liquid layer is $10 \mu \mathrm{m}$, the latter being initially situated at the center of the system. The initial thermodynamic conditions are $P_{0}=101,325 \mathrm{~Pa}, T_{0}=293.15 \mathrm{~K}$. At initial time, the left wall is heated to $T_{w}=373.15 \mathrm{~K}$, the right wall being insulated. After a transient evolution, a steady state establishes where the initial positions of the liquid-gas interfaces are recovered, due to mass conservation. In the gas, the density at steady state is unchanged, $T_{f}=T_{w}$ everywhere, and $P_{f}=P_{0} \cdot T_{f} / T_{0}=128,976.37 \mathrm{~Pa}$ following the perfect gas equation of state. This test case was treated in [10], based on a Arbitrary Lagrangian Eulerian (ALE) method (moving mesh). In this way, the interfaces between liquid and gas are real discontinuities, and there are no errors that could be attributed to the front tracking method and the existence of a mixing zone. Using an accurate discretization, we can consider the results given by this code as reference results (see [10] for more details about the ALE procedure).

The domain is discretized using 100 grid points. The time step is equal to $10^{-8} \mathrm{~s}$, due to stability limits. Fig. 8 presents trajectories of the two liquid-gas interfaces. The first remark that can be done is that those trajectories are parallel (the liquid has a rigid body motion), and that the initial positions are well recovered. This last point is a very important and demanding test for mass conservation for the method. 
A logarithmic representation of the interface trajectory reveals that in the early stages right after the heating of the left wall, an oscillatory motion of the liquid zone is observed. This effect is entirely due to the gas compressibility and was analyzed in detail in [10]. The corresponding thermodynamic pressure history is shown in Fig. 9, in linear and logarithmic scales. As the pressures in the two gaseous zones are not equal, the liquid undergoes an acceleration. Fig. 10 shows the fluid velocity in the whole domain, for two successive times. One can remark that the velocity is constant in the liquid, showing again that it behaves as a rigid body in this 1D case, whereas it is not the case in the gas due to compressibility effects. In the first microseconds, high velocities can be attained. The hydrodynamic pressure at $t=1 \mu \mathrm{s}$ is shown in Fig. 11 (left). The temperature fields for several successive times are shown in Fig. 11 (right) and reveal that after an initial transient where a piston effect can be observed in the rightmost part of the left gaseous zone [28], a purely conductive regime establishes in the left gaseous zone while temperature is nearly uniform in the right gaseous zone due to the adiabatic wall condition. The relative total mass variation in the cavity never exceeds $0.5 \%$ throughout the entire evolution, demonstrating good mass conservation properties of the algorithm.

Finally, in Fig. 12 we compare the results given by the front-tracking algorithm and the ALE approach, the latter being considered as reference. It can be seen, from the trajectories and the pressures history, that the results are very close, although the front-tracking algorithm is only first order in time. This validates the low Mach compressible/incompressible approach in the front-tracking framework.

\subsection{D numerical results}

\subsubsection{Case 1: a bubble in water in a heated box}

We now consider a two-phase system: a circular air bubble trapped in the middle of a hundred micron square container of water. The radius of the bubble is $30 \mu \mathrm{m}$. A uniform $32 \times 32$ grid is used. The initial characteristic $H$ function is presented in Figure 13. To focus on the phenomena of interest, buoyancy effects are neglected, and surface tension is not taken into account. Initially the fluid is at temperature $T_{0}=293.15 \mathrm{~K}$, and the thermodynamic pressure in air is $P_{0}=101325 \mathrm{~Pa}$. At initial time, the walls are heated at temperature $T_{1}=373.15 \mathrm{~K}$. In water, heating does not induce any flow. However, as soon as the heat flux becomes significant at the interface, the thermodynamic pressure increases in the air bubble. Some results are presented in Figures 14 and 15. The time variation of the mean pressure in Figure 15 clearly shows the mean pressure rise in the container as a result of the corresponding increase in the temperature of the air bubble. It is interesting to note the initially constant mean pressure during the phase of heat diffusion in the liquid. Figure 
15 also shows the evolution of the mean velocity modulus in time, which reaches a maximum value of the order of $10^{-6} \mathrm{~m} / \mathrm{s}$, justifying the low Mach flow hypothesis that was made. Figure 14 shows the velocity field superimposed on the temperature field and on the velocity divergence field at time equal $4 \mathrm{~s}$. The heat amount received by the bubble induces a compression flow inside of it. The water is not completely motionless as the air flow is not axisymmetric, due to the square shape of the cavity. Streamlines in the water show the development of eight vortices around the bubble (figure 14). After a transient period, steady state is reached, where temperature is at uniform value $T_{1}$ in the cavity, and by virtue of mass conservation the thermodynamic pressure in air satisfies $P_{1} / P_{0}=T_{1} / T_{0}=1.273$.

\subsubsection{Case 2 : two bubbles in water with initial thermodynamic pressure dif- ference}

We consider a $50 \mu m \times 100 \mu m$ rectangular closed box. The box walls are insulated. Two circular bubbles of air with initial radius $R_{1}=12 \mu \mathrm{m}$ and $R_{2}=12 \times \sqrt{1.4} \mu \mathrm{m}$ are included inside the liquid, centered at positions $(25 \mu \mathrm{m}, 25 \mu \mathrm{m})$ and $(25 \mu \mathrm{m}, 75 \mu \mathrm{m})$. The initial surface ratio of the bubbles $S_{2} / S_{1}$ is thus equal to 1.4. The fluid is initially at rest, and uniformly at a temperature $T_{0}=293.15 \mathrm{~K}$. At initial state, the two bubbles are at different thermodynamic pressures, $P_{1}=1.4 \mathrm{~atm}$ and $P_{2}=1 \mathrm{~atm}$. When time goes on, this initial pressure difference induces an oscillatory movement in the liquid, in the same way as in the above one dimensional case : the bubble at the higher pressure expands, the other shrinks, this being allowed by compressibility of the bubbles. As a consequence of the inertia of the incompressible fluid flow between the two bubbles, the pressure in first bubble decreases below the equilibrium pressure whereas that in the second bubble rises above equilibrium. This reverses the direction of the flow, and after that the bubbles undergo an oscillatory process involving alternate expansions and compressions. Mechanical equilibrium is observed in a short time delay, of about $t_{\text {mech }}=150 \mu \mathrm{s}$. Once the mechanical equilibrium is achieved, the system evolves very slowly towards steady-state due to the viscous and heat conduction effects. Indeed, the time period required to achieve thermal equilibrium is much longer due to the large time constant associated with heat transfer in the liquid phase. While the total area of gas should remain constant, the area of each bubble varies with expansions and compressions. At steady state, thermodynamic pressure $P_{f}$ and temperature $T_{f}$ are uniform. Let us denote by $M_{1}$ and $M_{2}$ the masses of gas in each bubble, that stay constant all over the evolution. Then we can write:

$$
\left\{\begin{array}{l}
M_{1}=\frac{P_{1}}{R T_{0}} S_{1}=\frac{P_{f}}{R T_{f}} S_{1 f} \\
M_{2}=\frac{P_{2}}{R T_{0}} S_{2}=\frac{P_{f}}{R T_{f}} S_{2 f}
\end{array}\right.
$$


$S_{1 f}$ and $S_{2 f}$ being the final surfaces of the bubbles, such that the final ratio of the surface of the bubbles is:

$$
\frac{S_{2 f}}{S_{1 f}}=\frac{M_{2}}{M_{1}}=\frac{P_{2}}{P_{1}} \cdot \frac{S_{2}}{S_{1}}
$$

In the same time, we can calculate the final pressure $P_{f}$ in the bubbles. The walls being adiabatic and rigid, the total energy in the box remains constant, implying that the final temperature $T_{f}$ is equal to $T_{0}$ as the total surface of gas and the mass of gas in each bubble is constant. Thus we can write:

$$
M_{1}+M_{2}=\frac{P_{f}}{R T_{0}}\left(S_{1}+S_{2}\right)
$$

yielding:

$$
P_{f}=\frac{P_{1} / P_{2}+S_{2} / S_{1}}{1+S_{2} / S_{1}} \cdot P_{2}
$$

In the present case we obtain $S_{2 f}=S_{1 f}$ and $P_{f}=\frac{7}{6} P_{2}=118212.5 \mathrm{~Pa}$ at steady state.

The results obtained using a $64 \times 128$ grid are shown in Fig. 16 and 17 . The characteristic function of the bubbles at several successive times is presented in Fig. 16. The variation of the shape and surface of the bubbles induced by the thermodynamic pressure variations is clearly seen here. In Fig. 17 are presented the time variations of several quantities, showing the damped oscillating motion of the system. Fig. 17-a) shows the surface ratio of the bubble, which should converge to 1 at steady state. An error of about $2 \%$ is visible. The thermodynamic pressures in the two bubbles, shown in Fig. 17-b), oscillate out of phase and converge to the same value after $0.15 \mathrm{~ms}$. In Fig. 17-c) one can see that the mean velocity modulus in the field reaches a value close to $1 \mathrm{~m} / \mathrm{s}$. Local velocities in the flow can reach $3 \mathrm{~m} / \mathrm{s}$, and very high accelerations are given to the fluid during the oscillatory period. Fig. 17-d) shows the normalized total gas area during time evolution, which should stay constant as the mass of gas is constant. One can notice that the mass conservation error remains very small all over the system evolution.

In order to quantify the grid convergence of the method, we have reproduced the same calculations for three different grids : $32 \times 64,64 \times 128$ and $128 \times 256$. Fig. 18 shows the time evolution of the surface ratio of the bubbles and the total gas area for the three grids. It can be noticed that the frequency of the oscillations slightly increases as the mesh is refined, and in the same time the damping time scale decreases. This effect could be related to the antidissipative nature of a first order time discretization. The solution on the middle mesh is close to that on the finer mesh, indicating that convergence is almost reached.

Knowing the exact steady values, we have calculated for each mesh the numerical error that is committed at steady state for the three quantities: $S_{2 f} / S_{1 f}$, 
Table 1

\begin{tabular}{cccc}
\hline Grid size & $S_{2 f} / S_{1 f}$ & Total gaseous area & $P_{f}$ \\
\hline $32 \times 64$ & 3.2 & 0.009 & 0.21 \\
\hline $64 \times 128$ & 1.6 & 0.007 & 0.067 \\
\hline $128 \times 256$ & 0.83 & 0.0001 & 0.022 \\
\hline
\end{tabular}

Convergence study : relative errors at steady state as a function of the grid size (in $\%)$

total gaseous area and $P_{f}$. The relative errors in percentage are compiled in Table 1. The quantity $S_{2 f} / S_{1 f}$ is seen to converge with first order accuracy, which is consistent with the first order accuracy in time of the scheme. However the convergence rate of the thermodynamic pressure is close to second order, while it is not regular for the total gaseous area. The reason why convergent rates are not the same for all variables remains unclear. The front tracking method also has some influence which is difficult to quantify precisely.

\subsubsection{Case 3 : six bubbles in water with initial thermodynamic pressure dif- ference}

We consider a $100 \mu m \times 150 \mu m$ square closed box. A uniform $128 \times 192$ grid is used. The box is filled with liquid water. Six identical circular air bubbles, $12 \mu \mathrm{m}$ in radius are regularly distributed in the liquid, as shown in Fig. 19 (left) which presents the initial field of the characteristic function $H$. The fluid is initially at rest with a uniform temperature $T_{0}=293.15 \mathrm{~K}$; different values of the thermodynamic pressure are imposed inside the bubbles as $P_{j 0}=1.4 \mathrm{~atm}$ for $j=1,4,6$ and $P_{j 0}=1$ atm for $j=2,3,5$, where the subscript $j$ represents the bubble's number and the subscript 0 stands for initial conditions (see Fig. 19 (left) for the numbering of the bubbles). At the very beginning of the transient process, the three bubbles with the highest pressures expand leading to the subsequent shrinkage of the three other bubbles. Mutual interactions then produce a complex multimodal oscillatory process until mechanical equilibrium is observed. This happens in a short time delay, of about $t_{m e c h}=100 \mu \mathrm{s}$. At that time, thermodynamic pressure is the same in all six bubbles as illustrated in Fig. 22 (right). Computed data show that at time $t_{\text {mech }}$, the temperatures within the bubbles are almost equal to $T_{0}$ and that only small superheats are noticeable in the liquid, close to the bubble's interfaces. Then combining in a first approximation, mass conservation and the equation of state for all six bubbles, one may estimate the thermodynamic pressure at time $t_{m e c h}$ :

$$
P_{f}=\frac{\sum_{j=1}^{6}\left(P_{j 0} S_{j 0}\right)}{\sum_{j=1}^{6} S_{j 0}}=\frac{\sum_{j=1}^{6}\left(P_{j 0}\right)}{6}=1.2 \mathrm{~atm}
$$


The bubble surface at time $t_{\text {mech }}$ reads:

$$
\frac{S_{j f}}{S_{j 0}}=\frac{P_{j 0}}{P_{f}} \quad \text { for } \quad \mathrm{j}=1,2, \ldots 6
$$

Numerical values are $\frac{S_{j f}}{S_{0}}=\frac{7}{6}$ for $j=1,4,6$ and $\frac{S_{j f}}{S_{0}}=\frac{5}{6}$ for $j=2,3,5$. Computed data presented in Fig. 22 are in close agreement with these estimated theoretical values.

The transient oscillating process (i.e. $t<t_{\text {mech }}$ ) is now discussed. In Fig. 19, the characteristic functions of the bubbles are presented for several successive times including $t_{m e c h}$. In addition, in Fig. 20 and 21 are also displayed for time $t=3 \mu \mathrm{s}$ the velocity field superimposed on the hydrodynamic pressure field, the extended thermodynamic pressure field as derived from Eq. (20) and the temperature field.

During the transient process, interactions between bubbles lead to deviations from the initial circular shape. The circular shape is however recovered at time $t_{m e c h}$ due to surface tension effects.

The hydrodynamic pressure $(\pi)$ presented in Fig. 20 and the extended thermodynamic $\left(P_{e}\right)$ pressure fields presented in Fig. 21 show, as expected, that these two quantities are very different in magnitude. Pressure $\pi$ is discontinuous at the bubble's interface due to surface tension, its value inside the bubbles being higher than in the liquid. Spatial variations of the hydrodynamic pressure are about $\rho_{l} V^{2}$ in magnitude. Concerning the extended thermodynamic pressure field $P_{e}$, it is reminded here that values inside the bubbles are the uniform thermodynamic pressures, their magnitude being about $10^{5} \mathrm{~Pa}$.

The velocity field, superimposed with the hydrodynamic pressure field in Fig. 20, reveals 2D complex flows in both the liquid and gaseous phases. One can remark that the velocity field is smooth in the vicinity of the interfaces, although we consider large density ratio and real surface tension effects. The liquid motion induced by the bubbles' expansion or compression may be associated, in the early stages, to a velocity as large as $1 \mathrm{~m} / \mathrm{s}$.

At time $3 \mu s$, temperature is uniform in the liquid, equal to the initial value $T_{0}$ due to the high thermal mass. On the other hand, the temperature inside the six bubbles is modified. Three bubbles, i.e. \#1, 4, 6 are cooled due to the expansion process, their respective temperatures being lower than the initial value $T_{0}=293 \mathrm{~K}$. In contrast, bubbles $\# 2,3,5$ are heated due to the work supplied by compression. Their temperatures are thus higher than the initial value. One will note that temperature in the bubbles is not uniform as time $t=3 \mu \mathrm{s}$ is smaller than the characteristic time scale for heat diffusion in the gas, estimated to be about $7 \mu \mathrm{s}$. 


\section{Conclusions and perspectives}

In this work, a single field model aimed at the numerical simulation of liquidgas flows has been proposed. The liquid phase is considered as strictly incompressible, while compressibility effects are taken into account in the gas through a low Mach approach. In contrast with previous works, our model allows for the complete mechanical and thermal description for the dynamics of each the two phases. The interface is treated using a front-tracking approach. In the case of multiple gaseous inclusions, the single field approach raises the problem of taking into account thermodynamic pressure differences effects. We propose a solution to this problem via the definition of an extended thermodynamic pressure field, which allows to recover the correct interface jump conditions. In addition, our method has the advantage of avoiding arithmetic precision problems that could be encountered if the thermodynamic pressure was incorporated into the hydrodynamic pressure field, the latter being several orders of magnitude smaller.

Concerning the numerical solution, the unsteady momentum equations are advanced in time through a prediction-projection time stepping algorithm. The projection step is done with a multigrid algorithm capable of dealing efficiently with large fluid density ratios. It allows us to enforce the divergence free condition in the liquid phase, and the specified value of the velocity divergence in the gas phase. The algorithm enforces exactly compatibility conditions and the coherence between energy and continuity equations at the discrete level. To illustrate the efficiency of our method, the Oscillatory Water Column isentropic test case was considered and comparisons were made with a single pressure (all Mach) method. We showed that the low Mach method allows the use of much larger time steps while maintaining time accuracy. Then the entire algorithm was first validated against a 1D reference non isothermal case. Calculations were next performed for $2 \mathrm{D}$ test cases where the flow is driven by thermopneumatic effects. Time evolution of the dynamics of the flow is discussed, showing that the whole configuration relaxes in an oscillatory way towards steady state. Alternate compression and dilatation phases result in corresponding heating and cooling of the gas within each bubble. In all cases, real fluids were considered (water and air), showing the robustness of the method with respect to the large density ratio involved.

Current work concerns the implicitation of the numerical method and its extension to second order accuracy in time. This should allow gain in CPU time, as the present explicit scheme is somewhat time consuming due to the restrictive diffusive stability condition. This is especially important as we plan to consider 3D applications, for which the optimization of the numerical method will be crucial. From the physical point of view, the model will be extended to take into account phase change. An important feature of our model is that, in contrast with purely incompressible approaches [17], it will give the possibility to account for the saturation curve, which links pressure and temperature at 
the interface for liquid-vapor flows.

\section{Acknowledgments}

The authors thank Damir Juric for providing his 2D incompressible front tracking code, and Olivier Daube and Yann Fraigneau for many fruitful discussions about projection methods and low Mach models.

\section{A. Multigrid solver for the Poisson equation}

The projection step consists in solving the equation

$$
\nabla \cdot(\lambda \nabla f)=s
$$

with homogeneous Neumann boundary conditions, where $\lambda=\frac{1}{\rho}$. For the twophase flow configurations at hand, the density $\rho$ is a discontinuous field with jumps of three orders of magnitude. Efficient solution of this type of equation has been the subject of intense research, as its solution can consume more then $80 \%$ of the total computing time, in particular when an explicit scheme is used for the prediction step. To this aim we use a multigrid solver along the lines of the algorithm proposed in $[27,21]$. It is not our purpose to discuss in detail the multigrid procedure which is well documented in several textbooks [2]. The ingredients of any multigrid procedure are the expression of the operator on the coarse grids, the relaxation scheme, the smoother, the coarse transfer operator that transfers the residual from a given grid to the next coarser grid and the fine transfer operator that transfers the correction from a grid to the next finer one to add it to the current estimate of the correction.

The computational domain $\Omega$ is covered with $N \times M$ cells of size $\Delta x_{i} \times \Delta z_{j}, i=$ $1, \cdots, N, j=1, \cdots, M . \lambda$ is defined by its values at the cell centers $\lambda_{i j}$. The solution is also sought at the cell centers. The finite volume discretization of the flux in (55) reads

$$
\lambda_{i+1 / 2, j} \frac{f_{i+1, j}-f_{i, j}}{x_{i+1, j}-x_{i, j}}
$$

at the east side of the cell with similar expressions for the other faces. Following $[27,21]$, the main idea of the algorithm is to enforce continuity of fluxes at the cell interfaces. It is well known that this is achieved by defining $\lambda_{i+1 / 2, j}$ as 
the harmonic average of $\lambda_{i+1, j}$ and $\lambda_{i, j}$ that is

$$
\frac{2}{\lambda_{i+1 / 2, j}}=\frac{1}{\lambda_{i+1, j}}+\frac{1}{\lambda_{i, j}}
$$

The coarse grids are defined by merging together two cells in each direction, which sets the cell boundaries as guidelines to define the coarse grids. The cell centers are defined accordingly. Once the grid is defined, we define $\lambda_{i j}$ on a grid from the weighted average of the 4 corresponding values on the next finer grid. Likewise, the residual restriction transfer operator is defined as the sum of the residuals of the four corresponding cells, which ensures conservativity of the mass unbalance.

The relaxation scheme is a simple SOR iteration whose acceleration parameter is adjusted empirically in order to produce the best convergence rate.

Concerning the interpolation operator for the correction of the error, it is also defined to ensure a constant flux interpolation in the following way: for each coarse cell, 8 constant flux interpolations are first produced from coarse cell neighbors, 4 at the middle of the faces, 4 at the corners. With straightforward notations,

$$
f_{e}=\frac{\lambda_{i+1, j} f_{i+1, j}+\lambda_{i, j} f_{i, j}}{\lambda_{i+1, j}+\lambda_{i, j}} ; f_{e n}=\frac{\lambda_{i+1, j+1} f_{i+1, j+1}+\lambda_{i, j} f_{i, j}}{\lambda_{i+1, j+1}+\lambda_{i, j}}
$$

with similar expressions for the other values. For each coarse cell, the corresponding 4 fine cell values are then defined as the arithmetic mean of the interpolated coarse values at the four corresponding corners.

In addition, for other application purposes, we define a solid phase indicator allowing for the presence of solid parts. One has then to solve the pressure equation with the homogeneous Neumann boundary conditions imposed at the fluid solid interface. The difficulty is to take into account this boundary condition to construct the operator in a systematic way. To do this, we recursively define the solid indicator of the coarse grids with the following principle : a coarse grid is considered as liquid iff one of the four corresponding fine cells is liquid. With all the ingredients, all operators can be recursively defined on all grids, allowing for an automatic algorithm once the various indicator functions are defined on the finest grid. 

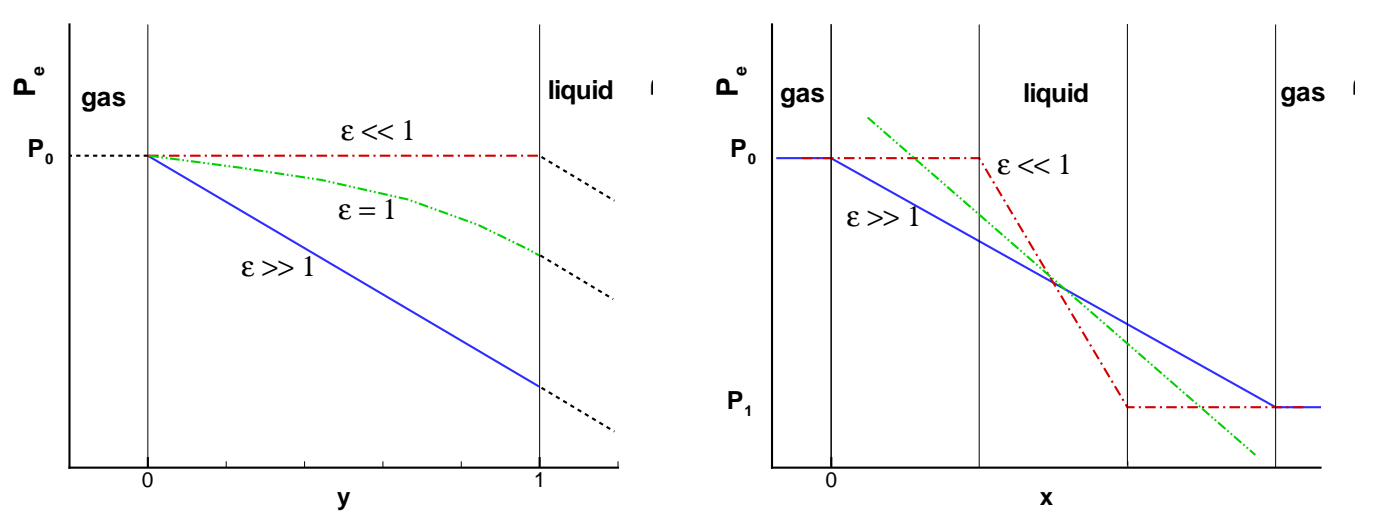

Fig. 1. Extended pressure profile. Left: inside a transition zone, for different values of $\epsilon$. Right: global for two gaseous zones (the green dash-dot-dot line shows the exact slope in the liquid).

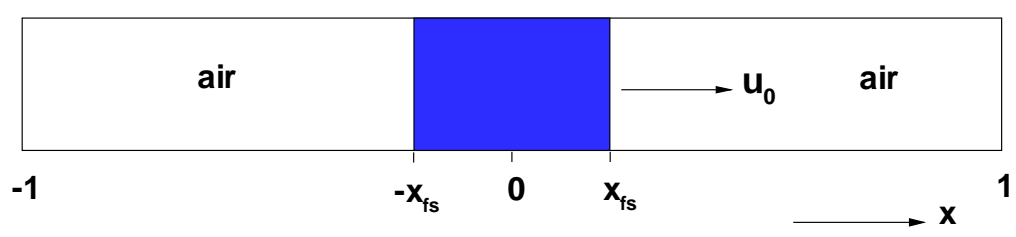

Fig. 2. Initial conditions for the Oscillatory Water Column test case.

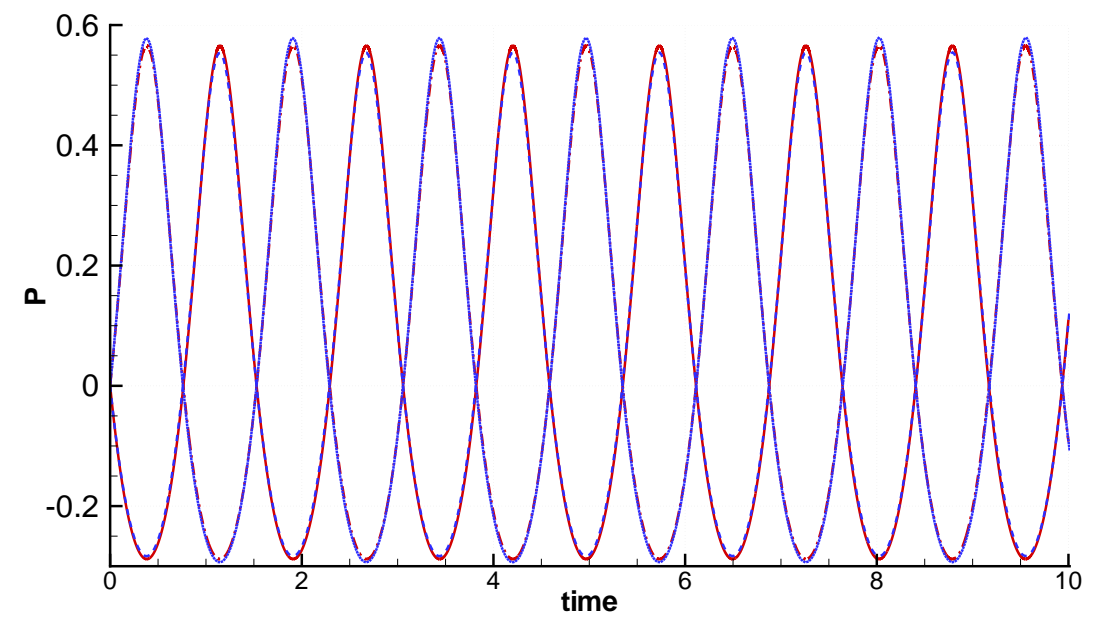

Fig. 3. Low Mach method. Time evolution of the pressure coefficients at left and right air columns (red solid and dash-dotted lines, left and right boundaries, $\mathrm{CFL}=1$, blue dashed and dotted lines, left and right boundaries, $\mathrm{CFL}=100$ ). 


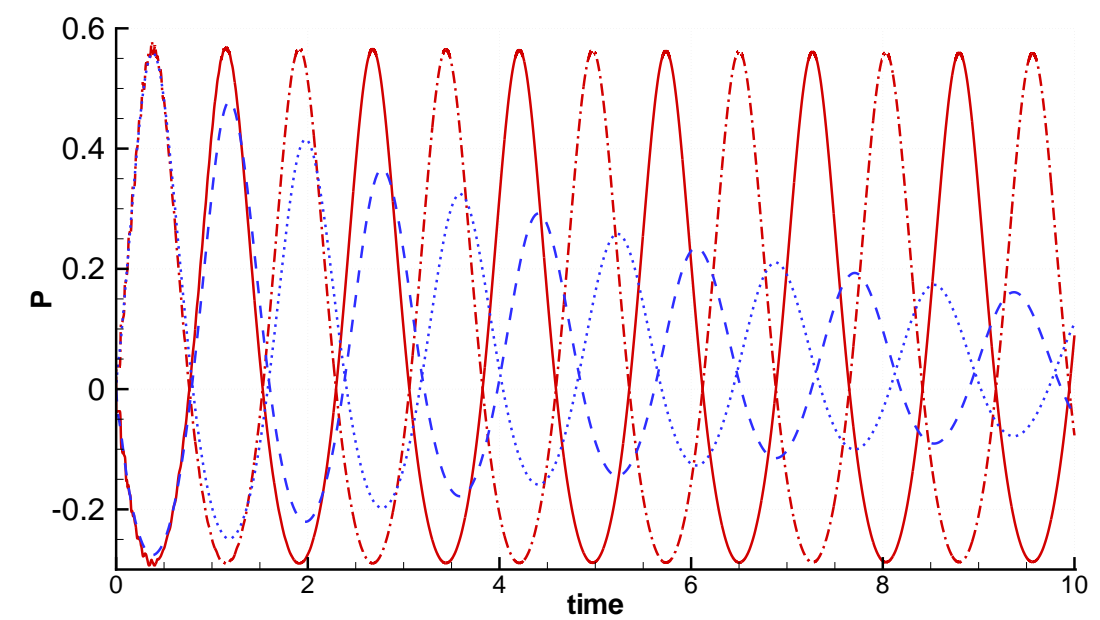

Fig. 4. Single pressure method. Time evolution of the pressure coefficients at left and right air columns (red solid and dash-dotted lines, left and right boundaries, $\mathrm{CFL}=1$, blue dashed and dotted lines, left and right boundaries, $\mathrm{CFL}=100$ ).

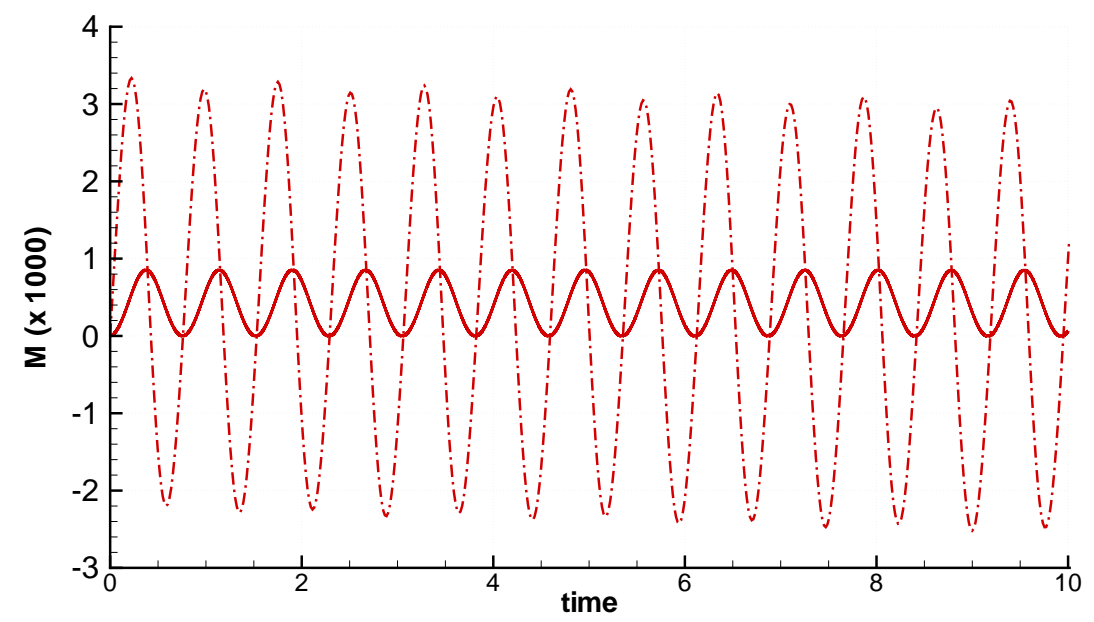

Fig. 5. Low Mach method. Time evolution of relative error in total mass of air. Solid line : $\mathrm{CFL}=1$, dash-dotted line $: \mathrm{CFL}=100$. 


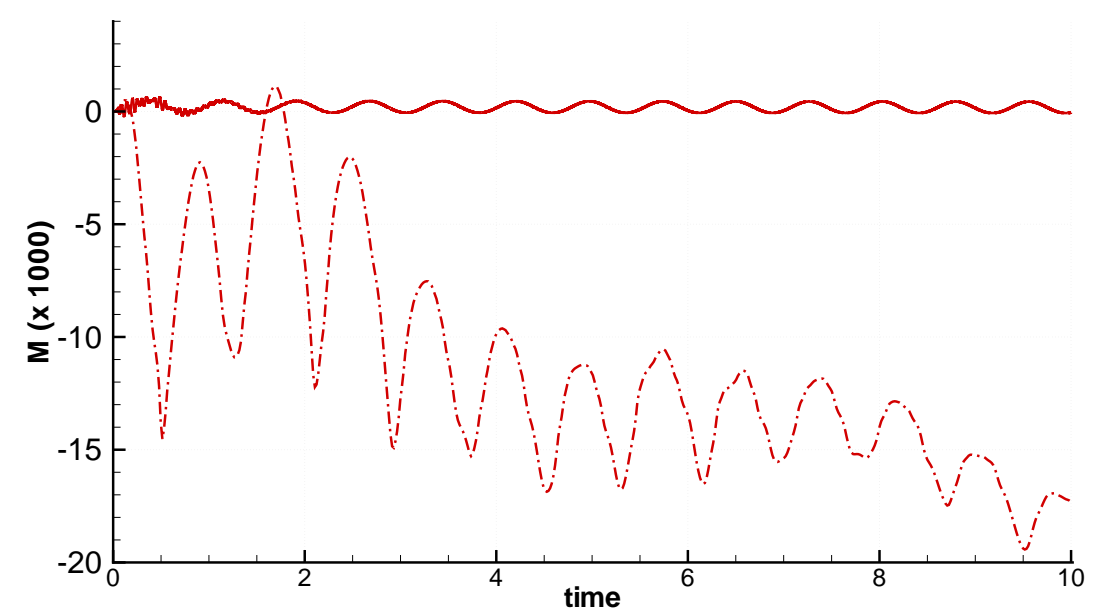

Fig. 6. Single pressure method. Time evolution of relative error in total mass of air. Solid line : $\mathrm{CFL}=1$, dash-dotted line : $\mathrm{CFL}=100$.

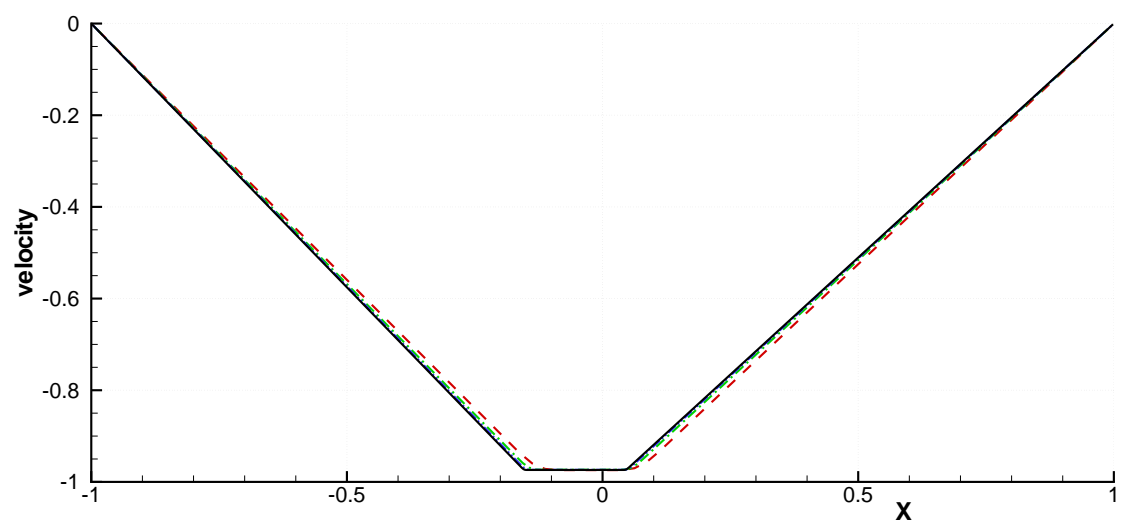

Fig. 7. Low Mach method, $C F L=100$. Grid convergence of the velocity field at time $t=10$. Solid black line $\delta x=1 / 320$, blue dash-dot-dotted $\delta x=1 / 160$, green dash-dot $\delta x=1 / 80$, red dashed $\delta x=1 / 40$.
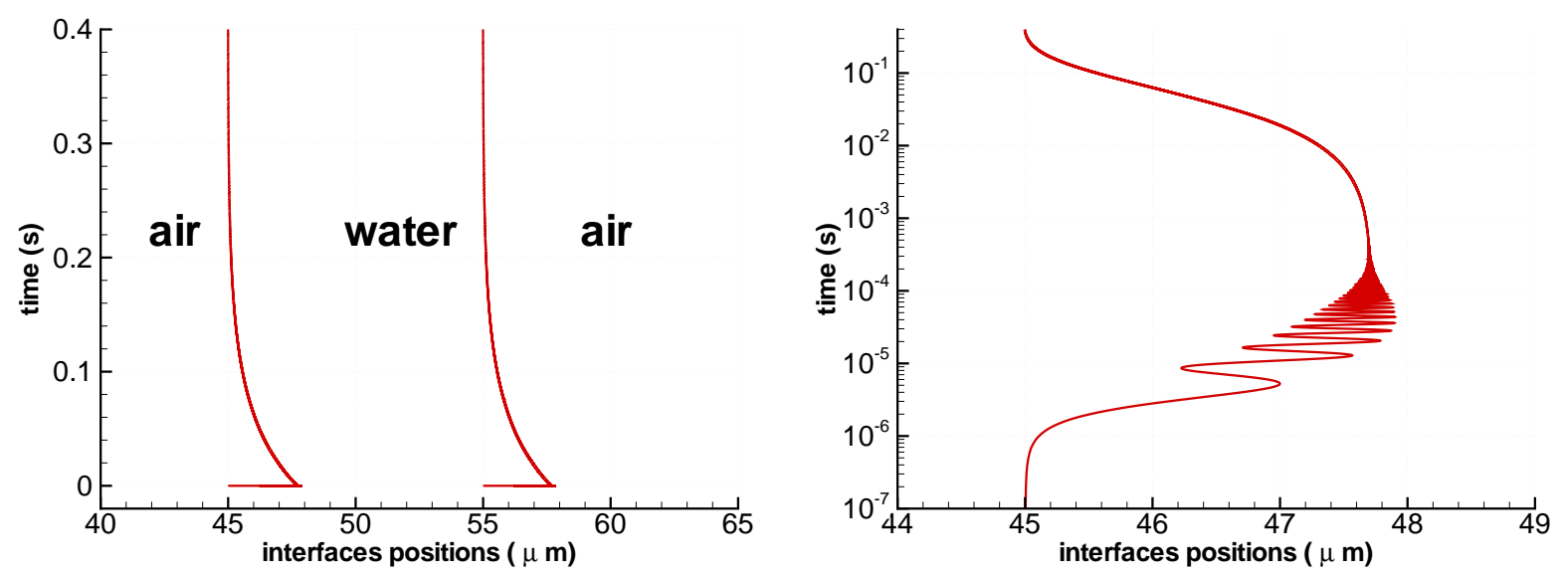

Fig. 8. Trajectories of interfaces, in natural time scale (left), log scale (right). 

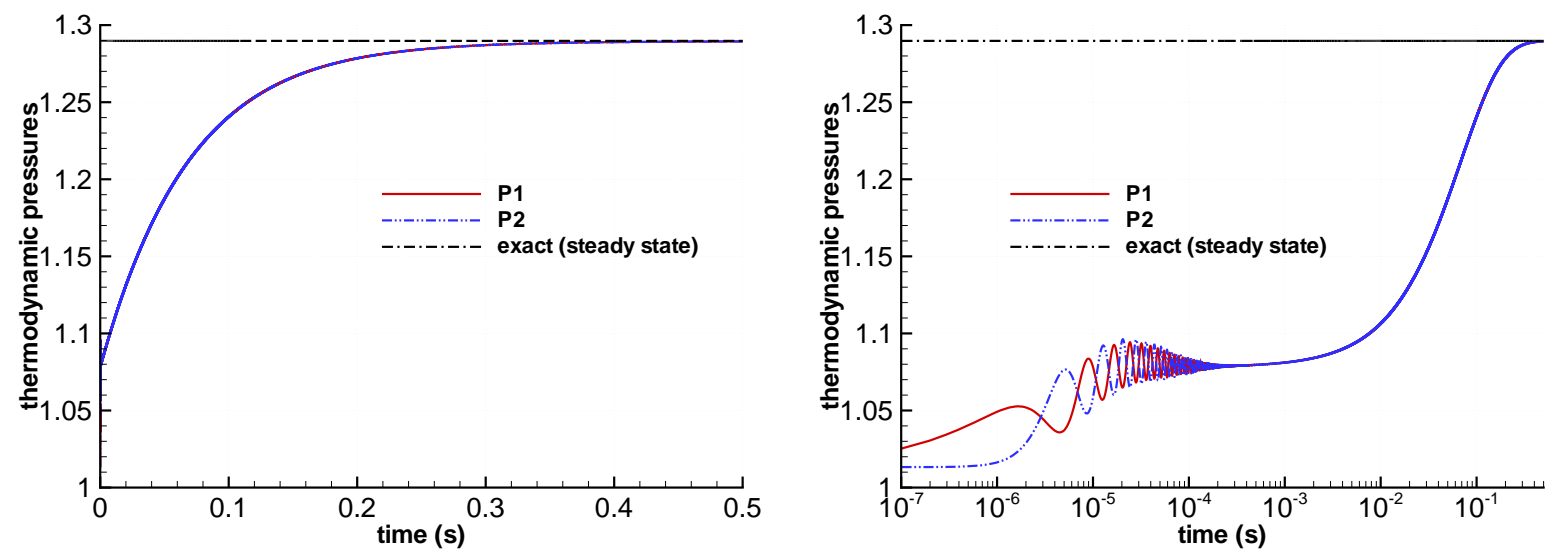

Fig. 9. Thermodynamic pressures (atm) in the gas zones, in natural time scale (left), $\log$ scale (right).
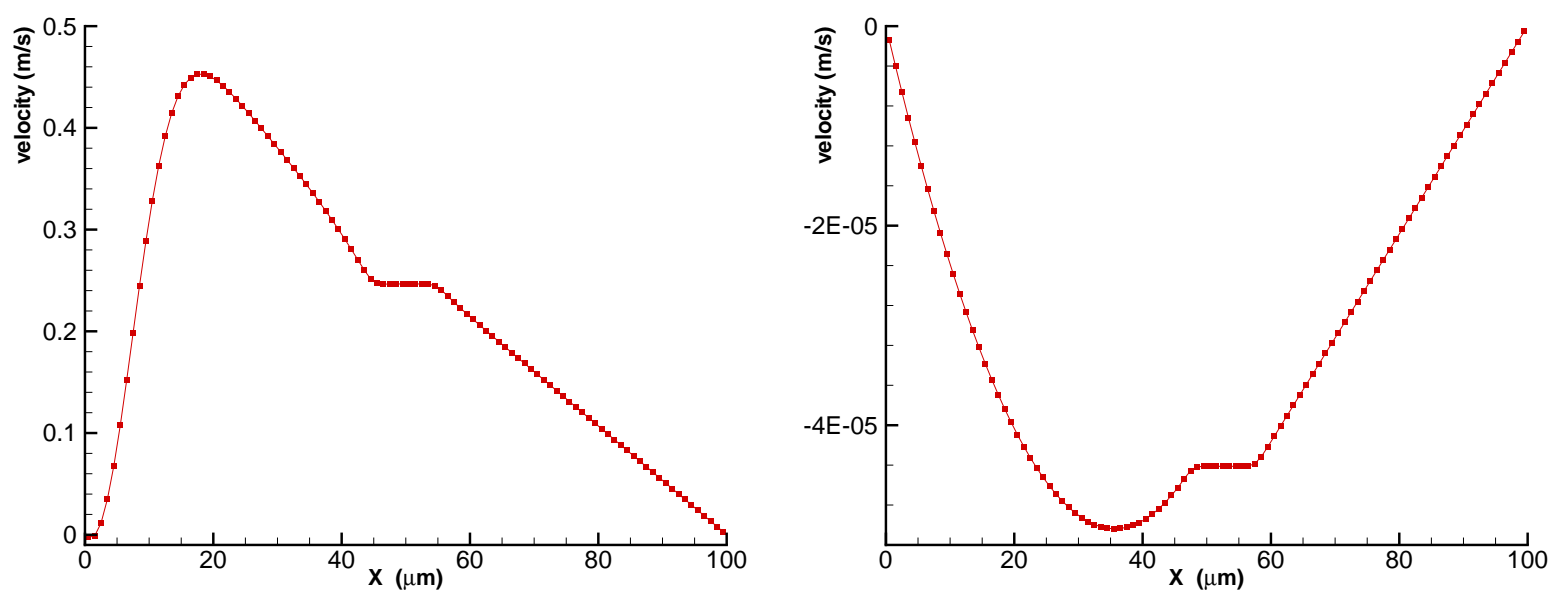

Fig. 10. Velocity in the domain, $t=1 \mu \mathrm{s}$ (left), $t=1 \mathrm{~ms}$ (right).
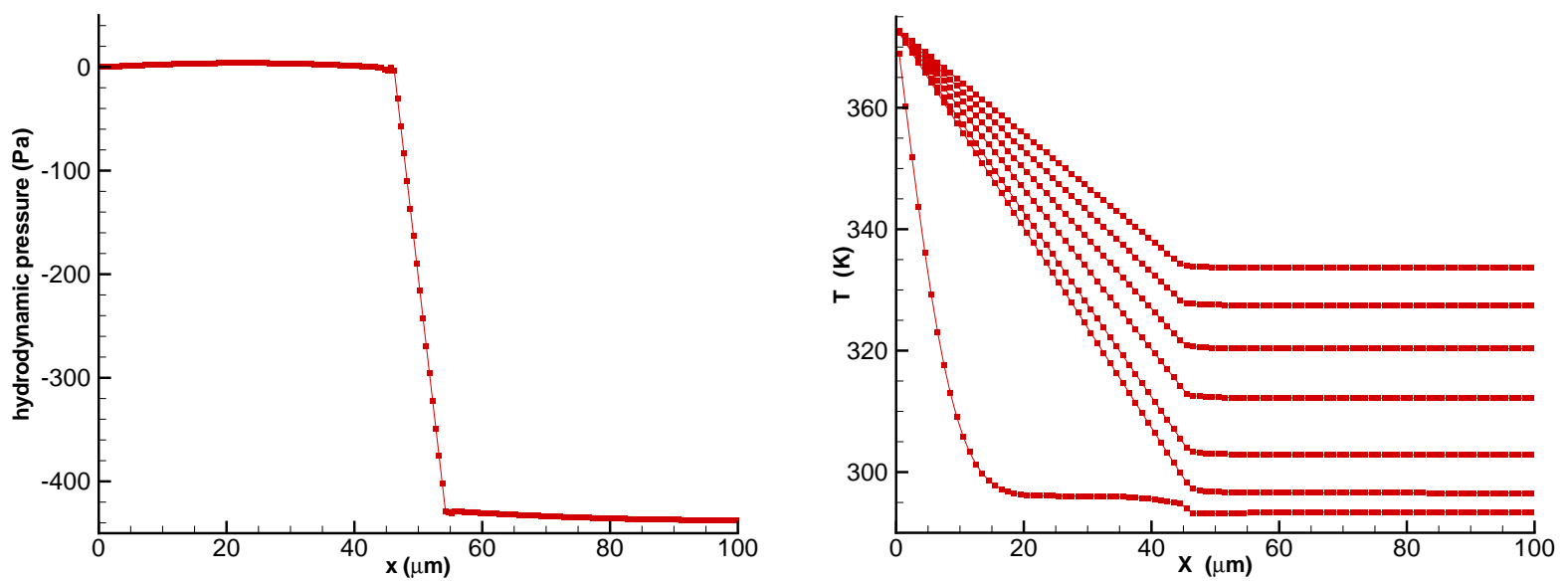

Fig. 11. Left : hydrodynamic pressure $(P a), t=1 \mu \mathrm{s}$. Right : temperature $(K)$, $t=1 \mu \mathrm{s}, t=4,5,6,7,8,9 \mathrm{~ms}$. 

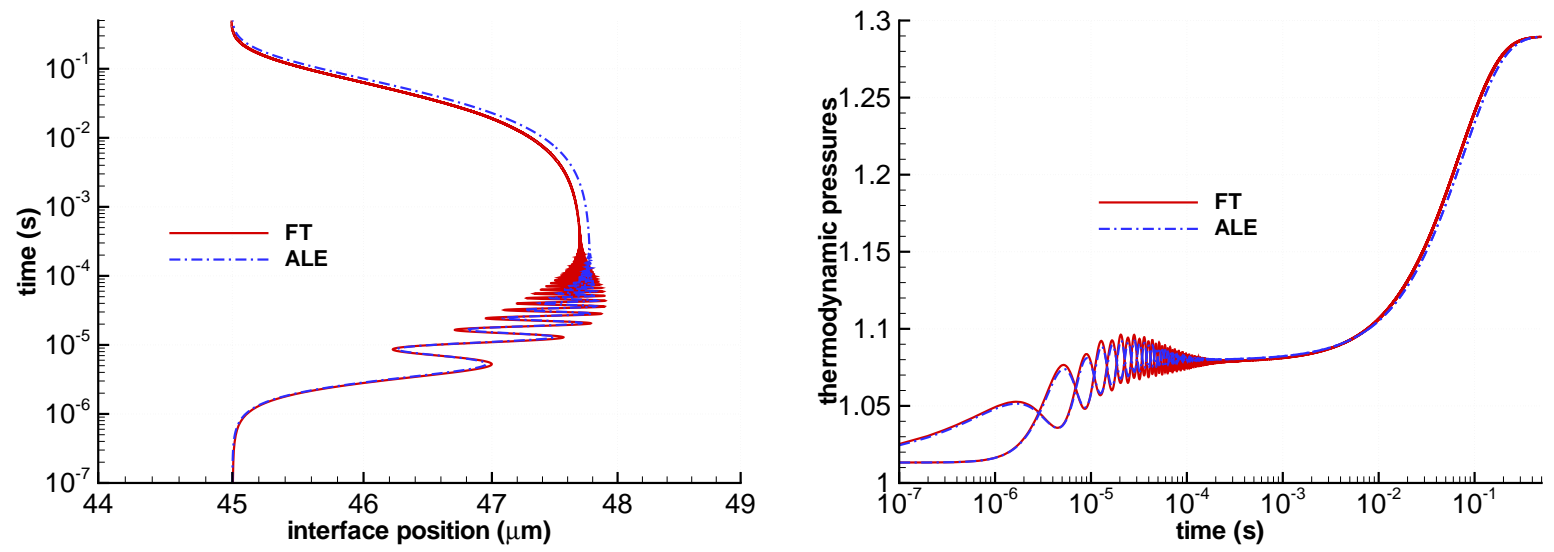

Fig. 12. Comparison of the front-tracking (FT) and ALE results. Left : left interface trajectory. Right : Thermodynamic pressures $(\mathrm{atm})$.

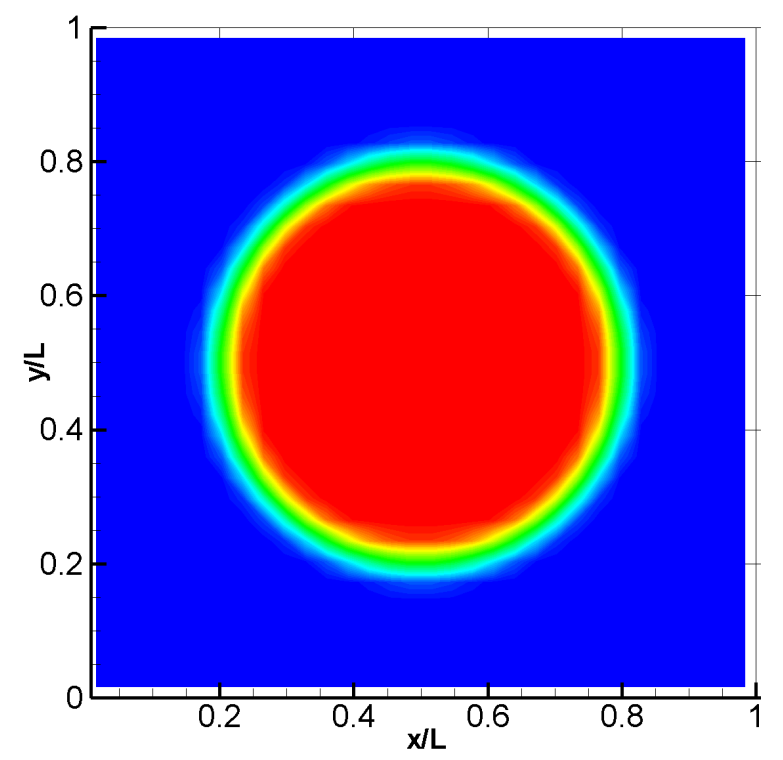

Fig. 13. Heated square box. Initial H function. Reference length $L=100 \mu m$. 

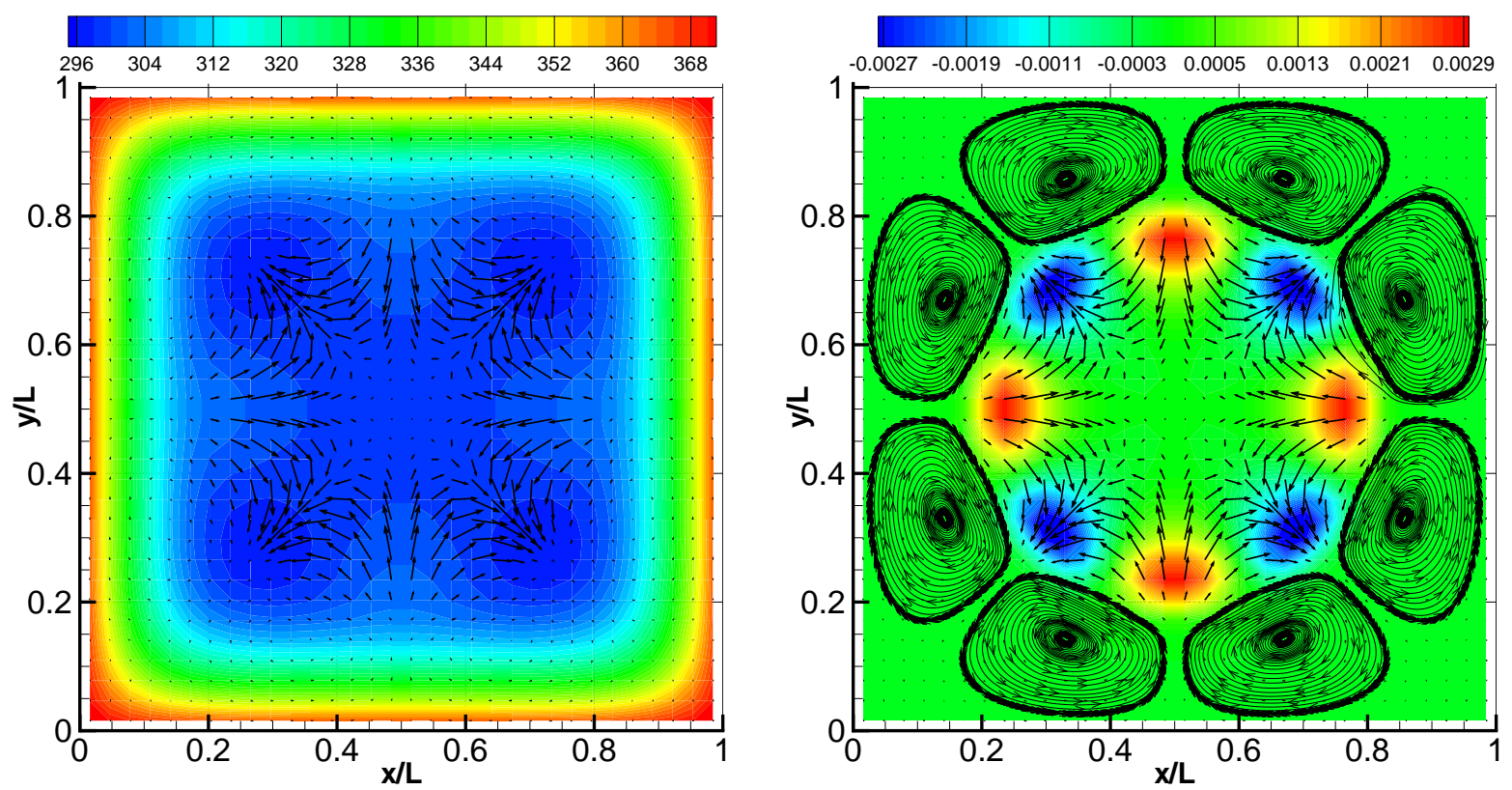

Fig. 14. Heated square box, $t=4 s$. Left : temperature and velocity fields. Right: divergence and velocity fields, streamlines superimposed in the liquid.
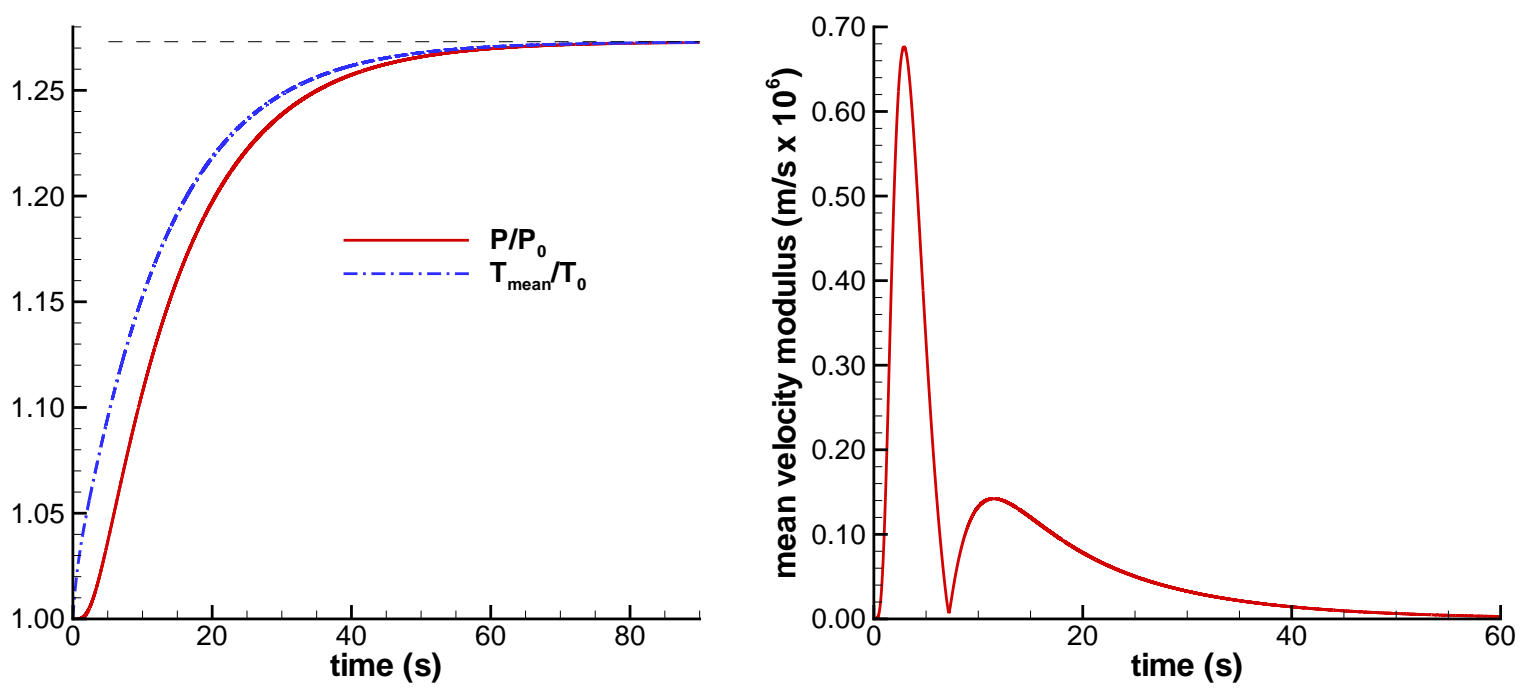

Fig. 15. Heated square box. Left : Normalized thermodynamic pressure in the bubble and mean temperature in the box versus time. The dashed straight line shows the steady exact value. Right : mean velocity modulus versus time.

\section{References}

[1] Bonometti, T., Magnaudet, J.: An interface-capturing method for incompressible two-phase flows. validation and application to bubble dynamics. Int. J. of Multiphase Flows 33, 109-133 (2007)

[2] Briggs, W.L., Henson, V.E., McCormick, S.F.: A Multigrid tutorial, 2nd edition edn. SIAM (2000) 

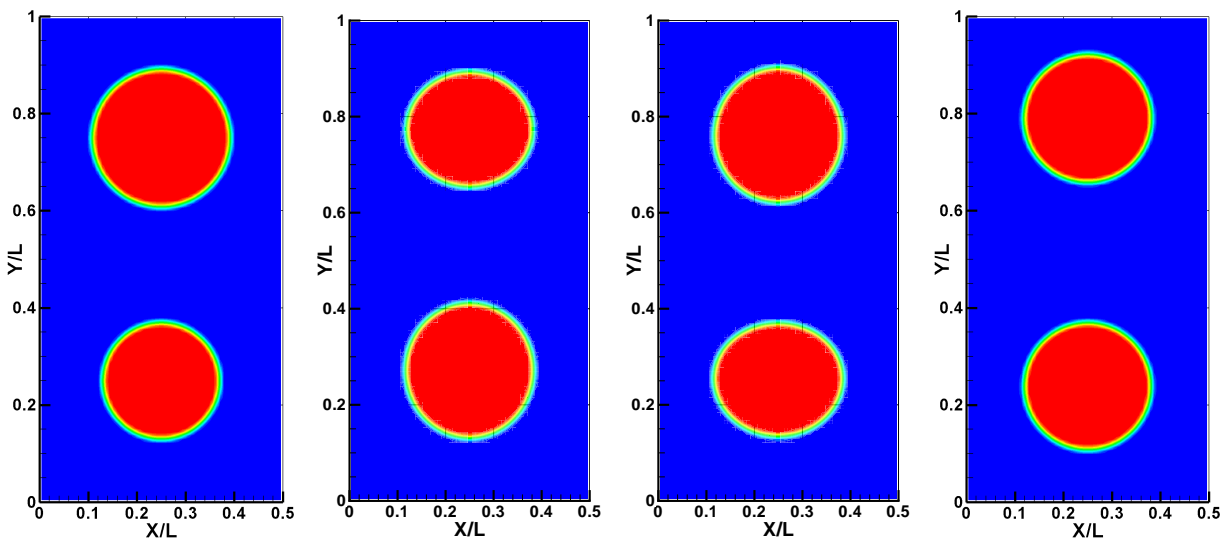

Fig. $16.64 \times 128$ grid. $H, t=0,4,8 \mu s$ and steady state. Reference length $L=100 \mu m$.

[3] Caboussat, A.: A numerical method for the simulation of free surface surface flows with surface tension. Computers and Fluids 35, 1205-1216 (2006)

[4] Caboussat, A., Picasso, M., Rappaz, J.: Numerical simulation of free surface incompressible liquid flows surrounded by compressible gas. J. Comput. Physics 203, 626-649 (2005)

[5] Caiden, R., Fedkiw, R.P., Anderson, C.: A numerical method for twophase flow consisting of separate compressible and incompressible regions. J. of Comput. Phys. 166, 1-27 (2001)

[6] Chenoweth, D., Paolucci, S.: Natural convection in an enclosed vertical air layer with large horizontal temperature differences. J. Fluid Mech. 169, 173-210 (1986)

[7] Daru, V., Duluc, M.C., Le Maître, O., Juric, D., Le Quéré, P.: Modélisation et simulation numérique du changement de phase liquidevapeur en cavité. CRAS Mécanique 224, 25-33 (2006)

[8] Delhaye, J. M.: Jump Conditions and Entropy Sources in Two-Phase Systems. Local Instant Formulation. Int. J. Multiphase Flow 1, 395-409 (1974)

[9] Dellacherie, S.: Numerical resolution of a potential diphasic low mach number system. J. Comput. Physics 223, 151-187 (2007)

[10] Duluc, M.C., Le Maître, O., Daru, V., Le Quéré, P.: Numerical study of liquid inclusion oscillations inside a closed 1-d microchannel filled with gas. Microfluidics and Nanofluidics 6, 163-177 (2009)

[11] Esmaeeli, A., Tryggvason, G.: Direct numerical simulations of bubbly flows. Part 1. Low Reynolds number arrays. J. Fluid Mech. 377, 313-345 (1998)

[12] Esmaeeli, A., Tryggvason, G.: Computations of film boiling. Part I: numerical method. Int. J. of Heat and Mass Transfer 47, 5451-5461 (2004)

[13] Forester, C., Emery, A.: A computational method for low mach number unsteady compressible free convective flows. J. Comput. Physics 10(3), 
a)

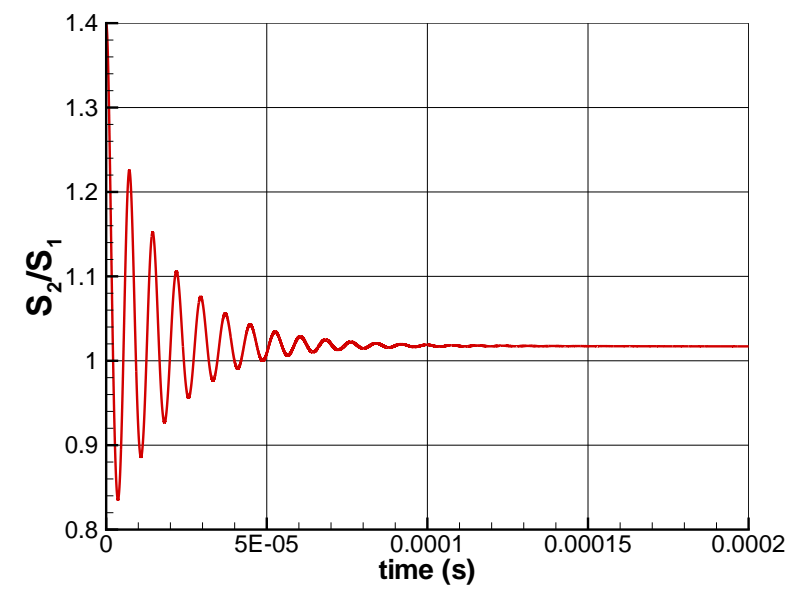

c)

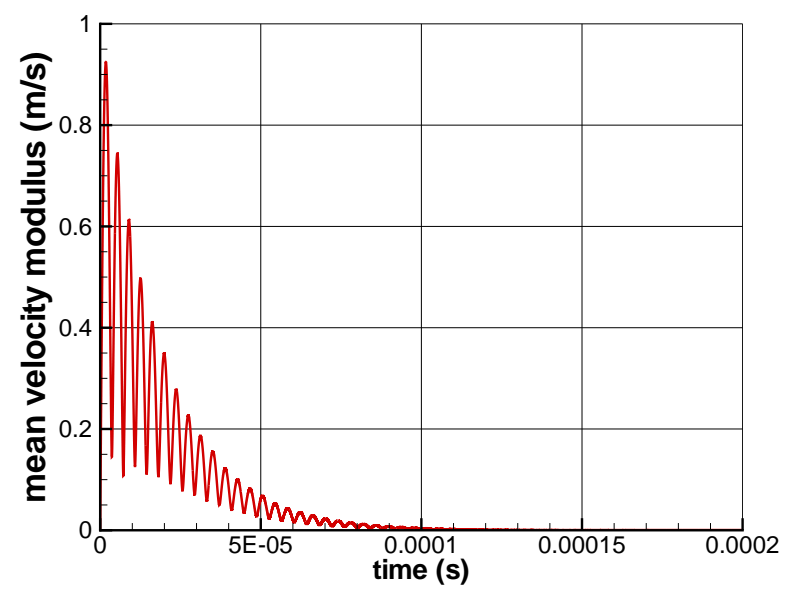

b)

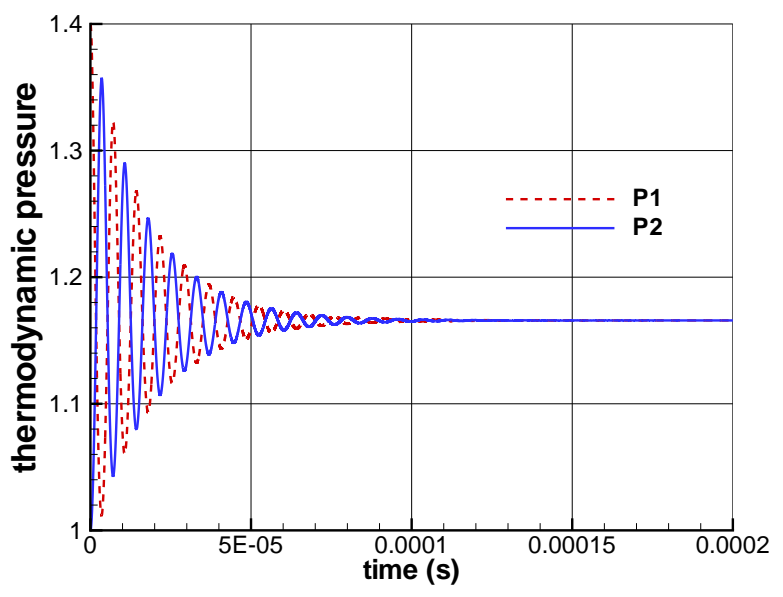

d)

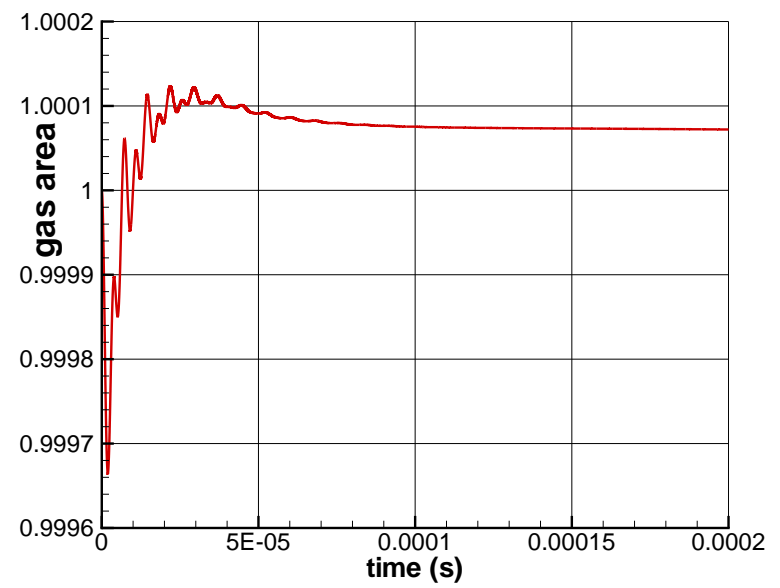

Fig. 17. Time evolution for the $64 \times 128$ grid. a): surface ratio of the bubbles, b): thermodynamic pressures $(\mathrm{atm}), \mathrm{c})$ : mean velocity modulus over the box, d) total bubbles area normalized by the initial area.

$$
\text { 487-502 (1972) }
$$

[14] Geng, X., Yuan, H., Og̃uz, N., Prosperetti, A.: The oscillation of gas bubbles in tubes: Experimental results. Journal of Acoustical Society of America 106(2), 674-681 (1999)

[15] Goda, K.: A multistep technique with implicit difference schemes for calculating two- or three-dimensional cavity flows. J. Comput. Phys. 30(1), 76-95 (1979)

[16] Ida M.: An improved unified solver for compressible and incompressible fluids involving free surfaces. II. Multi-time-step integration and applications. Computer Physics Comm. 150(3), 300-322 (2003)

[17] Juric, D., Tryggvason, G.: Computations of boiling flows. Int. J. Multiphase Flow 24(3), 387-410 (1998) 

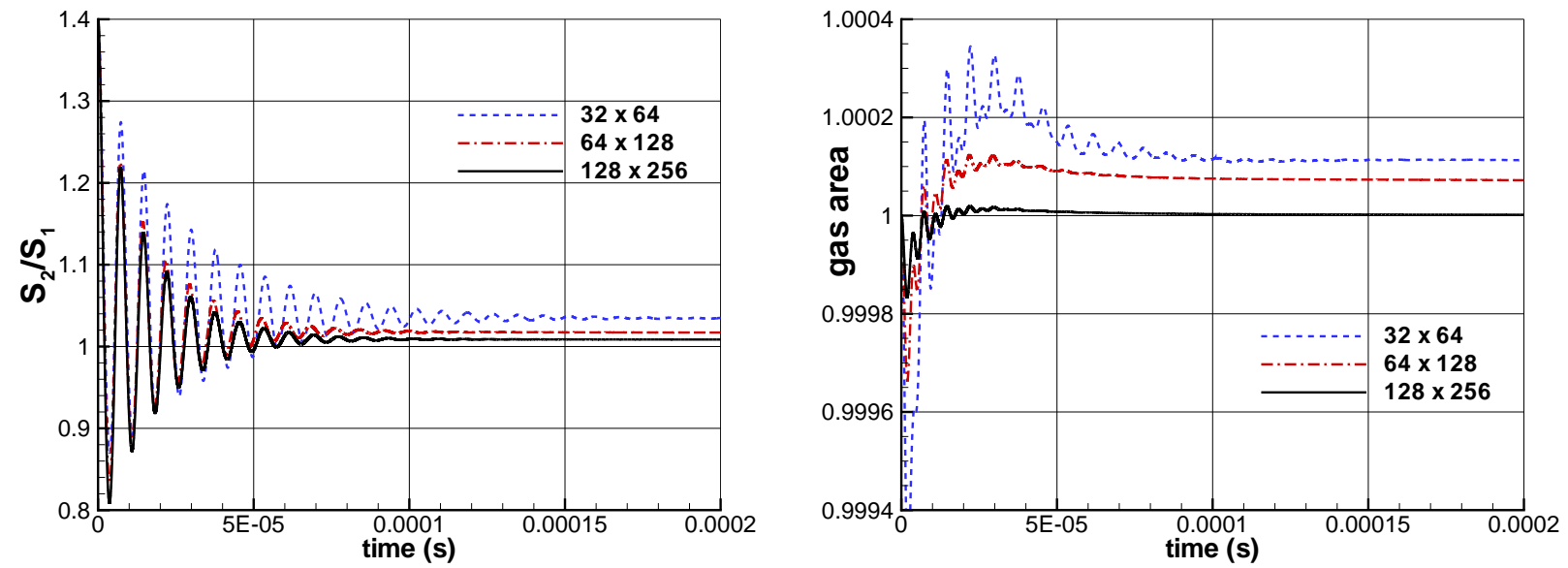

Fig. 18. Grid convergence of the bubbles surface ratio (left) and normalized gas area (right).
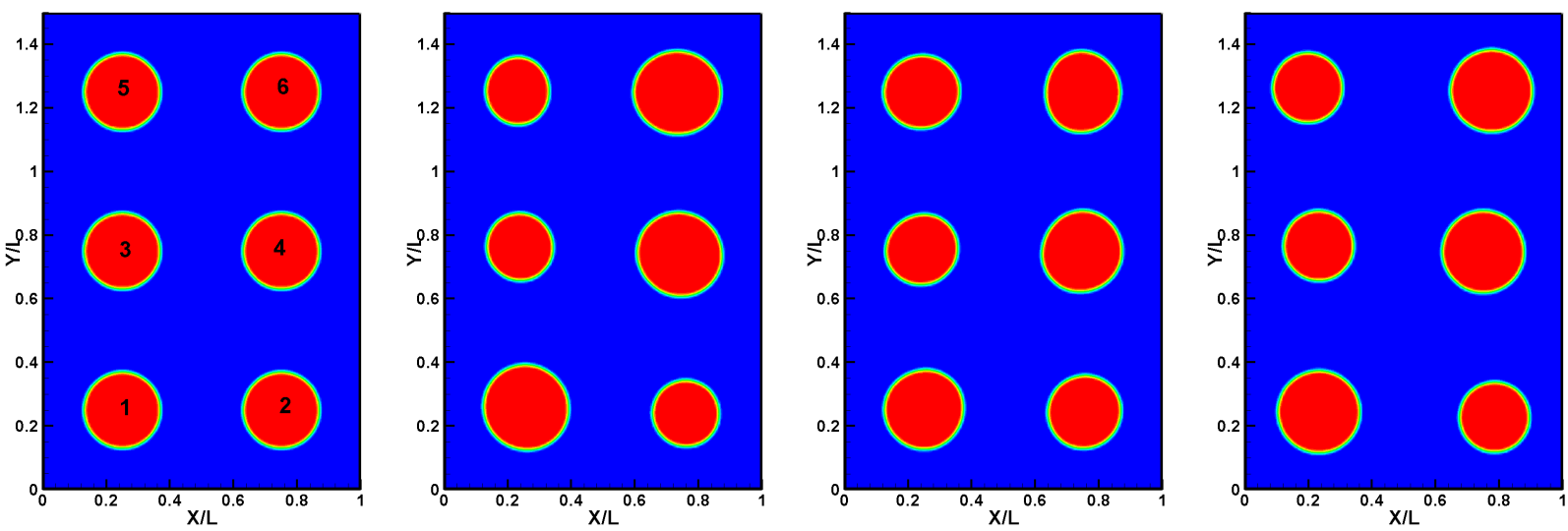

Fig. 19. Rectangular box with 6 bubbles at different initial thermodynamic pressures. From left to right : $H$ at times $0,3,6 \mu s$ and $t_{m e c h}$. Reference length $L=100 \mu m$.

[18] Kadioglu S. Y., Sussman M., Osher S., et al.: A second order primitive preconditioner for solving all speed multi-phase flows. J. Comput. Physics 209(2), 477-503 (2005)

[19] Koren B., Lewis M. R., van Brummelen E. H., van Leer B.: RiemannProblem and Level-Set Approaches for Homentropic Two-Fluid Flow Computations. J. Comput. Physics 181(11), 654-674 (2002)

[20] Kuszla, P., Daru, V.: Multifluid computation of droplets impact. In: Lecture Notes in Physics, vol. 515, pp. 524-529. C.H. Bruneau ed., Springer (1998)

[21] Kwak, D., Lee, J.: Multigrid algorithm for the cell-centered finite difference method ii: discontinuous coefficient case. Num. methods for partial differential equations 20(5), 742-764 (2004) 


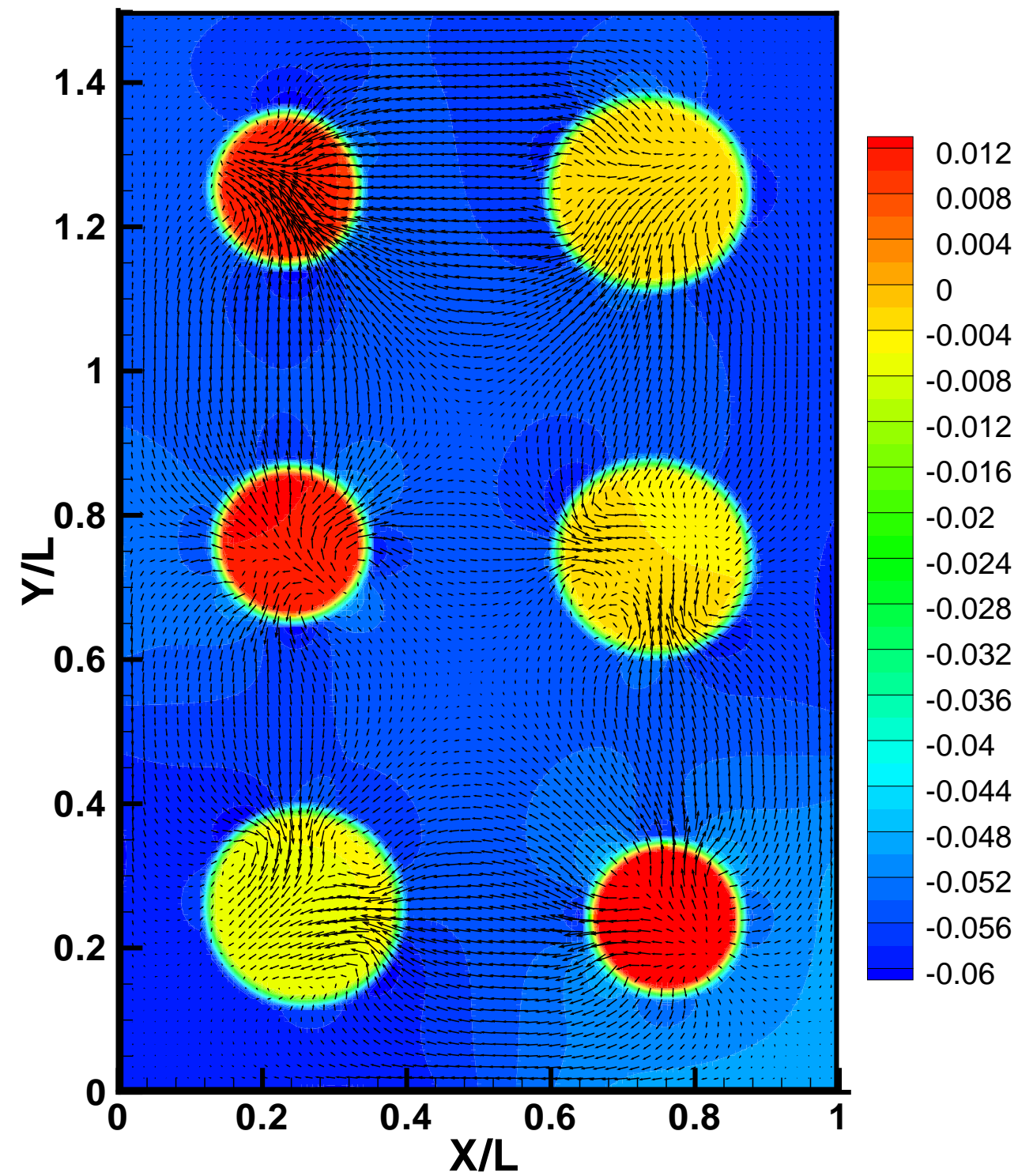

Fig. 20. Rectangular box with 6 bubbles at time $3 \mu s$. Hydrodynamic pressure $\pi$ $\left(\mathrm{Pa} / 10^{5}\right)$ with velocity field superimposed (only one vector out of four is shown). Reference length $L=100 \mu m$.

[22] Kwatra N., Su J., Gretarsson J. T., Fedkiw R.: A method for avoiding the acoustic time step restriction in compressible flow. J. Comput. Physics 228(11), 4146-4161 (2009)

[23] Lafaurie, B., Nardone, C., Scardovelli, R., Zaleski, S., Zanetti, G.: Modelling merging and fragmentation in multiphase flows with surfer. J. Comput. Phys. 113, 134-147 (1994)

[24] Le Quéré, P., Masson, R., Perrot, P.: A chebyshev collocation algorithm for 2d non-boussinesq convection. J. Comput. Physics 103(2), 320-335 (1992) 

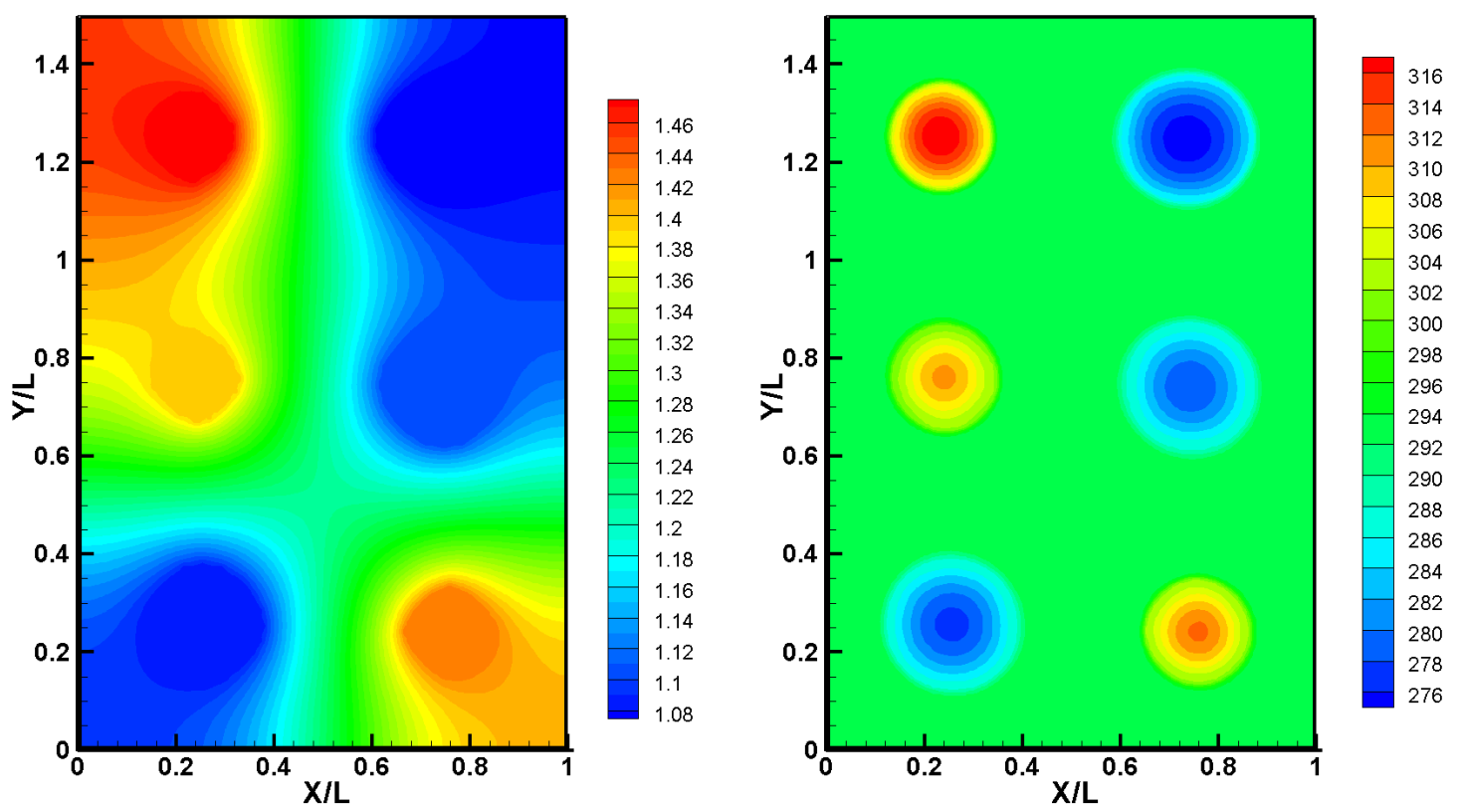

Fig. 21. Rectangular box with 6 bubbles at time $3 \mu$ s. Left: extended thermodynamic pressure $P_{e}\left(P a / 10^{5}\right)$, right: temperature $(K)$. Reference length $L=100 \mu m$.
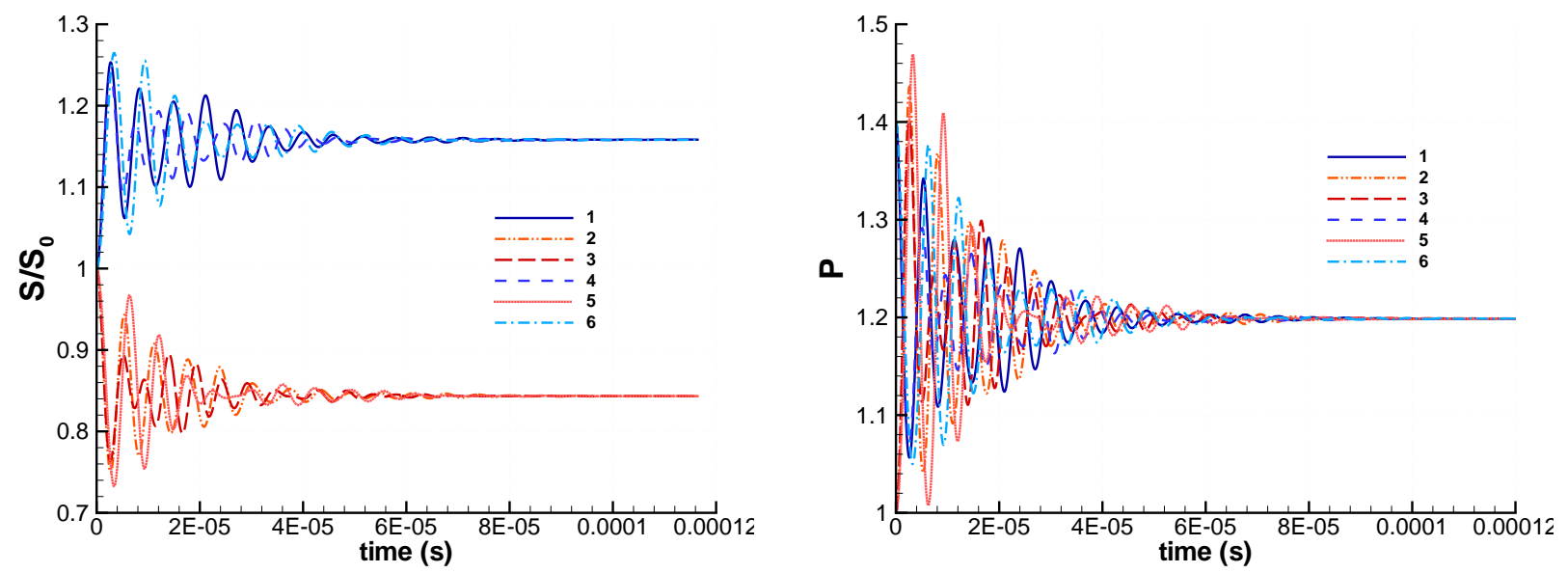

Fig. 22. Time evolution of the surface (left) and thermodynamic pressure (right) of the six bubbles.

[25] Le Quéré, P., Weisman, C., Paillère, H., Vierendeels, J., Dick, E., Becker, R., Braack, M., Locke, J.: Modelling of natural convection flows with large temperature differences. Part 1 : Reference solutions. Math. Modelling and Num. Analysis 39(3), 609-616 (2005)

[26] Legendre, D., Borée, J., Magnaudet, J.: Thermal and dynamic evolution of a spherical bubble moving steadily in a superheated or subcooled liquid. Phys. of Fluids 10, 1256-1272 (1998)

[27] Liu, C., Liu, Z., McCormick, S.: An efficient multigrid scheme for elliptic equations with discontinuous coefficients. Comm. in Applied Num. Meth. 8, 621-631 (1992) 
[28] Masuda, Y., Aizawa, T., Kanakubo, M., Saito, N., Ikushima, Y.: Numerical simulation of two-dimensional piston effect and natural convection in a square cavity heated from one side. International Communications in Heat and Mass Transfer 31(2), 151-160 (2004)

[29] Ménard, T., Tanguy, S., Berlemont, A.: Coupling level set/vof/ghost fluid methods: Validation and application to $3 \mathrm{~d}$ simulation of the primary break-up of a liquid jet. Int. Journal of Multiphase Flow 33, 510-524 (2007)

[30] Og̃uz, N., Prosperetti, A.: The natural frequency of oscillation of gas bubbles in tubes. Journal of Acoustical Society of America 103(6), 33013308 (1998)

[31] Ory, E., Yuan, H., Prosperetti, A., Popinet, S., Zaleski, S.: Growth and collapse of a vapour bubble in a narrow tube. Physics of fluids 12(6), 1268-1277 (2000)

[32] Paillère, H., Le Quéré, P., Weisman, C., Vierendeels, J., Dick, E.: Modelling of natural convection flows with large temperature differences. Part 2 : Contributions. Math. Modelling and Num. Analysis 39(3), 617-621 (2005)

[33] Paolucci, S.: Sandia National lab. Report SAND 82-8257. Unpublished (1982)

[34] Peskin, C.S.: Numerical analysis of blood flow in the heart. J. Comput. Physics 25, 220-252 (1977)

[35] Prosperetti, A., Tryggvason, G.: Computational Methods for Multiphase Flows. Cambridge University Press (2007)

[36] Saurel, R., Abgrall, R.: A multiphase godunov method for compressible multifluid and multiphase flows. J. Comput. Phys. 150, 425-467 (1999)

[37] Scardovelli, R., Zaleski, S.: Direct numerical simulation of free surface and interfacial flow. Ann. Rev. Fluid Mech. 31, 567-603 (1999)

[38] Sethian, J.: Level Set Methods and Fast Marching Methods. Cambridge University Press (1999)

[39] Shin, S., Abdel-Khalik, S., Daru, V., Juric, D.: Accurate representation of surface tension using the level contour reconstruction method. J. Comput. Physics 203(2), 493-516 (2005)

[40] Shin, S., Juric, D.: Modeling three-dimensional multiphase flow using a level contour reconstruction method for front-tracking without connectivity. Journal of Computational Physics 180, 427-470 (2002)

[41] Shyue, K.M.: An efficient shock-capturing algorithm for compressible multicomponent problems. J. Comput. Phys. 142, 208-242 (1998)

[42] Sussman, M., Smereka, P., Osher, S.: A level set approach for computing solutions in incompressible two-phase flows. J. Comput. Phys. 114, 146159 (1994)

[43] Tanguy, S., Berlemont, A.: Application of a level set method for simulation of droplet collisions. Int. Journal of Multiphase Flow 31, 1015-1035 (2005)

[44] Tryggvason, G., Bunner, B., Esmaeeli, A., Juric, D., Al-Rawahi, N., 
Tauber, W., Han, J., Nas, S., Jan, Y.: A front-tracking method for the computations of multiphase flow. J. Comput. Physics 169(2), 708-759 (2001)

[45] Xiao F.: Unified formulation for compressible and incompressible flows by using multi-integrated moments I: one-dimensional inviscid compressible flow. J. Comput. Physics 195(2), 629-654 (2004)

[46] Xiao F., Akoh R., Ii S.: Unified formulation for compressible and incompressible flows by using multi-integrated moments II: Multi-dimensional version for compressible and incompressible flows. J. Comput. Physics 213(1), 31-56 (2006)

[47] Yabe T., Xiao F., Utsumi T.: The constrained interpolation profile method for multiphase analysis. J. Comput. Physics 169 (2), 556-593 (2001)

[48] Yin, Z., Prosperetti, A.: A microfluidic 'blinking bubble' pump. Journal of Micromechanical Microengineering 15, 643-651 (2005) 